Cite this: Phys. Chem. Chem. Phys., 2014, 16, 7037

Received 3rd January 2014, Accepted 21st February 2014

DOI: $10.1039 / c 4 c p 00029 c$

www.rsc.org/pccp

\section{Central-transition double-quantum sideband NMR spectroscopy of half-integer quadrupolar nuclei: estimating internuclear distances and probing clusters within multi-spin networks $\dagger$}

\begin{abstract}
Andreas Brinkmann ${ }^{\star a}$ and Mattias Edén ${ }^{b}$
We introduce a strategy to estimate the size of clusters of recoupled homonuclear half-integer quadrupolar nuclei under magic-angle spinning (MAS) conditions, by combining double-quantum (2Q) sideband NMR experiments with an approximate numerical analysis based on the summation of all spinpairs present over a given radius of the structure. The experiment relies solely on the evolution of homonuclear $2 \mathrm{Q}$ coherences (2QC) among the central-transitions (CT) of half-integer spins and is suitable for probing clusters in network structures, such as those encountered in large groups of oxidebased materials. Experimental ${ }^{11} \mathrm{~B},{ }^{23} \mathrm{Na}$ and ${ }^{27} \mathrm{Al} \mathrm{NMR}$ results are presented on bis(catecholato)diboron, $\mathrm{Na}_{2} \mathrm{SO}_{4}$ and $\mathrm{Al}_{2} \mathrm{O}_{3}$, respectively; in each case, the growth of the spin-cluster size was monitored from a series of experiments that employed progressively lengthened 2QC excitation intervals. Our new approach is the first option for probing larger constellations of half-integer spins; it provides similar information as the "multiple-quantum spin counting" experiment, which is well-established for spin-1/2 applications but has hitherto not been demonstrated for half-integer spins undergoing MAS. We also discuss various options for determining the internuclear distance within a (nearly) isolated pair of halfinteger spins by comparing the experimental $2 Q$ sideband NMR spectra with results from numerical simulations involving various degrees of approximation.
\end{abstract}

\section{Introduction}

Precise interatomic-distance information constitutes the core of a detailed understanding of a given molecular structure, and is generally best determined by diffraction methods. However, whenever the possibility of obtaining large and well-ordered crystals is not feasible, either because the structure lacks longrange order or feature dynamics within its sub-units, solid state nuclear magnetic resonance (NMR) spectroscopy offers an attractive alternative. The power of high-resolution magic-angle spinning (MAS) NMR that utilizes distance-dependent throughspace dipolar interactions restored by "dipolar recoupling", techniques ${ }^{1-5}$ is well documented for providing highly accurate information about selected internuclear distances ${ }^{4,6}$ and dihedral angles $^{7-12}$ in well-defined clusters of spin-1/2 nuclei-primarily involving ${ }^{1} \mathrm{H},{ }^{13} \mathrm{C}$ and ${ }^{15} \mathrm{~N}$ in bio-molecules. This is generally

\footnotetext{
${ }^{a}$ Measurement Science and Standards, National Research Council Canada, 1200 Montreal Road, M-40, Ottawa, Ontario K1A OR6, Canada. E-mail: Andreas.Brinkmann@nrc-cnrc.gc.ca

${ }^{b}$ Physical Chemistry Division, Department of Materials and Environmental Chemistry, Arrhenius Laboratory, Stockholm University, 10691 Stockholm, Sweden $\dagger$ Electronic supplementary information (ESI) available. See DOI: 10.1039/ c4cp00029c
}

arranged by site-specific isotopic labeling, which is feasible thanks to the combination of well-established synthetic routes and the low natural abundance of ${ }^{13} \mathrm{C}$ and ${ }^{15} \mathrm{~N}$. Recent progress in dipolar recoupling NMR methodology is gradually also permitting the simultaneous accurate extraction of increased numbers of internuclear distances in uniformly $\left({ }^{13} \mathrm{C},{ }^{15} \mathrm{~N}\right)$ enriched biological macromolecules. ${ }^{4}$

Yet, comparatively few distance-determinations involving homonuclear (i.e., of the same species) spins-1/2 are demonstrated in the large groups of inorganic network structures, e.g., minerals, ceramics, glasses and porous materials, where the nature of the structures implies very large networks of mutually coupled spins. Further, the state-of-affairs is much worse when considering even semi-quantitative internuclear distance-estimates between half-integer spin quadrupolar nuclei, ${ }^{13-15}$ despite a strong impetus for exploiting them for structural studies by NMR, stemming from their dominating abundance in inorganic materials. ${ }^{14,16}$ Owing to their multi Zeeman-level character and the presence of strong quadrupolar interactions, the development of homonuclear dipolar recoupling methodology targeting half-integer spins is a complex task, as reviewed in ref. 14 and 15. Yet, during the past decade, several alternatives have been proposed. ${ }^{15,17-31}$ However, these recoupling techniques, 
including dipolar self-recoupling under $\mathrm{MAS}^{32,33}$ and experimentation on non-spinning samples, ${ }^{34-36}$ have predominantly been applied for gaining qualitative information about connectivities, proximities, and distributions of homonuclear spins, or relative orientations of NMR interaction tensors, ${ }^{15,17-19,22-28,30,31,37-44}$ whereas present reports on direct internuclear distance-estimates remains very sparse..$^{20,21,45,46}$ Furthermore, recent work exploits homonuclear through-bond (" $J ")$ interactions among half-integer spins to gain information about site-connectivities in inorganic structures. $^{47-49}$

All existing strategies to directly measure internuclear distances via their associated dipolar interactions within homonuclear pairs of half-integer spins undergoing MAS, have relied on double-quantum (2Q) dipolar recoupling radio-frequency (rf) pulse sequences. The magnetization may either (i) be allowed to evolve under their resulting effective dipolar Hamiltonian, ${ }^{20,21}$ or be converted into $2 \mathrm{Q}$ coherences (2QC) among the central transitions (CTs) of the two spins, onwards referred to as " $2 \mathrm{Q}_{\mathrm{CT}}$ ". The latter option has involved either (ii) monitoring of the $2 \mathrm{Q}_{\mathrm{CT}}$ generation (which is directly dependent on the dipolar interaction-strength) for prolonged excitation intervals $\left(\tau_{\text {exc }}\right),{ }^{45}$ or (iii) using 2Q-1Q 2D correlation spectroscopy, which results in an internuclear distance-sensitive spinning sideband manifold if the $t_{1}$-evolution is sampled faster than at completed rotational periods. ${ }^{46}$ Strategy (iii) was introduced by Spiess and co-workers as a general means of determining NMR interactionparameters in systems involving at least one spin-1/2.6,50-54 All three approaches to interatomic distance-measurements have been applied extensively in the area of spins-1/2, which possesses a very substantial arsenal of 2Q-recoupling schemes (reviewed in ref. 1-4), whereas much fewer such options exist for half-integer spins. ${ }^{15,19,22,24,26-31}$

In ref. 46, we introduced CT 2Q sideband NMR spectroscopy to the realm of half-integer spins: we demonstrated its potential by employing a simple analysis for extracting the internuclear distance in the pair of ${ }^{11} \mathrm{~B}$ spins in bis(catecholato)-diboron (with a relative uncertainty of $2 \%$ ), in excellent accordance with the value obtained by single crystal X-ray diffraction (XRD). ${ }^{55}$ Herein, we refine and justify this distance-estimate by accounting for effects from longer-range intermolecular dipolar interactions, by employing numerically exact simulations involving up to four coupled ${ }^{11} \mathrm{~B}$ ( $\operatorname{spin} I=3 / 2$ ).

Yet, isolated pairs of quadrupolar spins are rarely encountered in inorganic systems. We therefore also explore the CT 2Q NMR sideband technique further in the context of the densely coupled networks of ${ }^{23} \mathrm{Na}(I=3 / 2)$ and ${ }^{27} \mathrm{Al}(I=5 / 2)$ in anhydrous sodium sulfate $\left(\mathrm{Na}_{2} \mathrm{SO}_{4}\right)$ and $\alpha-\mathrm{Al}_{2} \mathrm{O}_{3}$, respectively. We demonstrate that a recently introduced general strategy for modeling the NMR spectrum from a multi-spin system-that approximates the intractable exact calculation of the large spinsystem by a summation of the responses from each spin-pair in the structure over a given radius ${ }^{56}$ - successfully reproduces the experimental $2 \mathrm{Q}_{\mathrm{CT}}$ spinning sideband manifold from each of the two model structures. The sideband envelope depends on the product of the dipolar coupling constant $\left(b_{j k}\right)$ and the 2QC reconversion interval $\left(\tau_{\text {rec }}\right)$ employed following the $2 Q_{\mathrm{CT}}$ evolution. ${ }^{6,50-54}$
Hence, given that a series of experiments is recorded for increasing $\tau_{\text {exc }}=\tau_{\text {rec }}$, and the known atomic coordinates of the structure are used to calculate the 2D NMR spectrum for each $\tau_{\text {exc }}$-value, our new method constitutes an alternative to existing "spin-counting" NMR techniques ${ }^{57-64}$ for determining the relationship between the cluster-size of mutually interacting spins and the recoupling interval $\tau_{\text {exc }}$.

\section{Double-quantum sideband NMR spectroscopy}

\subsection{Experimental protocol}

Fig. 1 displays the rf pulse scheme for the CT 2Q sideband NMR experiment. It starts by enhancing the spectral signal-to-noise ratio by increasing the population-difference across the CT: ${ }^{39}$ here, we employ a smoothly attenuated single frequency sweep, ${ }^{46,65}$ but any alternative technique is equally applicable, e.g., those of ref. 66-69. The longitudinal magnetization is subsequently converted into $2 \mathrm{Q}_{\mathrm{CT}}$, in this work by repeating a $\mathrm{R} 2{ }_{2}^{1} \mathrm{R} 2{ }_{2}^{-1}$ 2Q-recoupling sequence ${ }^{26} m$ times to arrange a 2QC excitation interval of $\tau_{\text {exc }}=4 m \tau_{\mathrm{r}}$, where the sample rotational period $\tau_{\mathrm{r}}$ relates to the angular spinning frequency $\omega_{\mathrm{r}}$ as $\tau_{\mathrm{r}}=2 \pi / \omega_{\mathrm{r}}$. This pulse scheme conforms to the R-symmetry class, ${ }^{2,3,70}$ and requires that the spin- $I$ CT nutation frequency $\omega_{\text {nut }}^{\mathrm{CT}}=(I+1 / 2)\left|\gamma_{I}\right| B_{1} / 2$ equals $\omega_{\text {nut }}^{\mathrm{CT}}=\omega_{\mathrm{r}} / 2$, where $\gamma_{I}$ is the spin gyromagnetic ratio and $B_{1}$ the $\mathrm{rf}$ amplitude. All recoupling pulses are sandwiched between two CT-selective $\pi / 2$-pulses, each of duration $\tau_{90}^{\text {sel }}$, as discussed in detail in ref. 26, 27 and 29. While we recommend using the $\mathrm{R} 2{ }_{2}^{1} \mathrm{R} 2{ }_{2}^{-1}$ scheme for $2 \mathrm{Q}_{\mathrm{CT}}$-excitation in samples displaying relatively low resonance-frequency dispersions, ${ }^{27,29}$ the CT 2Q sideband experiment may utilize any $2 \mathrm{Q}_{\mathrm{CT}}$ generator for half-integer spins, ${ }^{2,24,27-31}$ or supercycles thereof. The pulse trains described in ref. 27, 29 and 30 are beneficial for cases featuring large frequency-spreads among the recoupled spin sites. Next follows a Hahn spin-echo of duration $T$, timed such

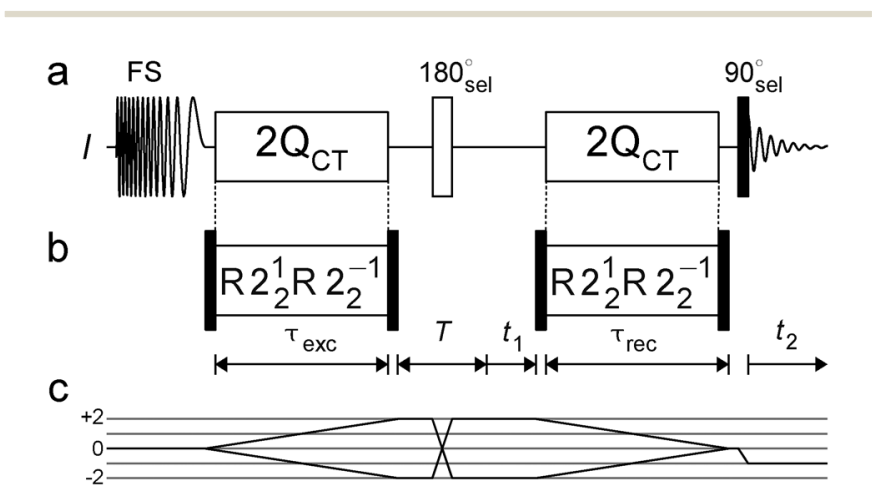

Fig. 1 (a) General radiofrequency pulse sequence to record two-dimensional homonuclear double-quantum correlation spectra on half-integer quadrupolar nuclei for the purpose of $2 Q_{C T}$ sideband spectroscopy. The block labeled " $2 Q_{C T}$ " represents any pulse sequence generating central transition $2 \mathrm{Q}$ coherences. A frequency sweep (FS) may be used to enhance the central transition population difference. (b) The pulse sequence $R 2{ }_{2}^{1} R 2_{2}^{-1}$ (ref. 26) is specifically used in this work for $2 Q_{C T}$ excitation. The subscript "sel" indicates central-transition-selective pulses. (c) Coherence transfer pathway diagram ${ }^{71}$ for the /-spins. 
that $T+2 \tau_{90}^{\mathrm{sel}}=p \tau_{\mathrm{r}}$, where $p$ is any even integer; the phasecycling $^{71}$ of the CT-selective $\pi$-pulse should retain solely the $\pm 2 \mathrm{Q}_{\mathrm{CT}} \rightarrow \mp 2 \mathrm{Q}_{\mathrm{CT}}$ pathways to block $2 \mathrm{QC}$ involving the satellite transitions (STs), ${ }^{24}$ as indicated in Fig. 1c.

In the absence of $t_{1}$-evolution, the 2Q $\mathrm{Q}_{\mathrm{CT}}$ coherences are transferred directly into detectable CT single-quantum coherences (1QC) by repeating the $\pi / 2$-sandwiched $\mathrm{R} 2{ }_{2}^{1} \mathrm{R} 2{ }_{2}^{-1}$ sequence for a reconversion interval $\tau_{\text {rec }}$, followed by a CT-selective $\pi / 2$-pulse (Fig. 1a). While one may arrange that $\tau_{\text {exc }} \neq \tau_{\text {rec, }}{ }^{72}$ herein we employ equal excitation and reconversion segments throughout all our experiments. However, when $t_{1}>0$, the incrementation of the evolution interval has strong bearings on the 2Q-1Q 2D NMR spectrum. When internuclear proximity-information is desired, $t_{1}$ is normally sampled at integer multiples of $\tau_{\mathrm{r}}{ }^{24,26-28,30,39,41,56}$ which ensures that the rotor position is identical at the start of each $\tau_{\text {exc }}$ and $\tau_{\text {rec }}$ event, thereby forcing all spinning sidebands to fold onto each respective centerband ("isotropic") frequency: the $2 \mathrm{D}$ spectral coordinates then reveals all unique $2 \mathrm{Q}_{\mathrm{CT}}$ frequencies $\omega_{j k}^{2 \mathrm{Q}}$ along the indirect (vertical) dimension, correlated with their respective CT 1QC frequencies $\omega_{j}$ and $\omega_{k}$ that appear along the direct (horizontal) spectral dimension.

However, if the $t_{1}$-incrementation is performed in steps smaller than $\tau_{\mathrm{r}}$, a spinning sideband formation occurs along the indirect spectral dimension. It typically originates from two distinct mechanisms: (i) Rotor-modulation, stemming from $2 \mathrm{Q}_{\mathrm{CT}}$-evolution under anisotropic interactions, produces sidebands at positions shifted by $p \omega_{\mathrm{r}}$ ( $p$ is any integer) from the centerband frequency. They are well-known and become significant in all MAS NMR experimentation when the magnitude(s) of the anisotropic interaction(s) exceed the spinning frequency. (ii) Rotor-encoded spinning sidebands, on the other hand, only appear at odd integer multiples of $\omega_{\mathrm{r}}$, and arise if the spatial sample position is different at the start of each 2QC excitation and reconversion segment. ${ }^{6,50-54}$ These sideband-intensities are essentially independent on the relative sizes of the MAS frequency and the chemical shift and quadrupolar anisotropies active during the evolution interval $t_{1}$, provided that rotormodulated contributions are eliminated by employing fast MAS. However, the precise appearance of the rotor-encoded sideband-envelope depends on the product $b_{j k} \tau_{\text {exc }}=b_{j k} \tau_{\text {rec }}$, which provides a route to measure the dipolar coupling constant $b_{j k},{ }^{6,50-54}$ related as $r_{j k}{ }^{-3}$ to the internuclear separation $r_{j k}$ between spins $j$ and $k$. We exploit this feature to estimate internuclear distances between half-integer spins, ${ }^{46}$ as explored further herein. There are two options for implementing the $t_{1}$-sampling in Fig. 1a, which have bearings both on the experimental time and the precise numerical protocol to extract information from the rotor-encoded sidebands:

(1) The protocol in Fig. 1a is used to record a complete 2Q-1Q 2D NMR spectrum by sampling the evolution period over a ms-range, i.e., over many rotational periods of the sample. This is required to achieve sufficient signal-resolution in the presence of several distinct spin-pairs in the structure.

(2) The evolution interval is only sampled over the first rotational period, i.e., at $N$ time-points $t_{1}^{j}=j \tau_{\mathrm{r}} / N$, with $j=0$, $1, \ldots, N-1$, which in practice is implemented as an array of
$N$ 1D NMR experiments. This approach is only beneficial when the sample produces one unique $2 \mathrm{Q}_{\mathrm{CT}}$ frequency, but is then highly advantageous for reducing the experimental time (as well as that for the numerical simulations discussed below), as the 2Q $\mathrm{Q}_{\mathrm{CT}}$ spinning sideband manifold results directly on Fourier transformation (with respect to $t_{1}$ ) of the "pseudo-2D" data-set $\left\{S\left(t_{1}^{j}, t_{2}=0\right)\right\}$; these values are in practice obtained by integrating the total intensity along the $\omega_{2}$ spectral dimension for each consecutive $t_{1}^{j}$-value. Similar approaches have been utilized both for numerical simulations of MAS NMR experiments (e.g., see ref. 73), and in several experimental NMR protocols, such as for spin-counting ${ }^{57-64}$ and molecular torsion-angle determinations. $^{7}$

Implementation (1) was used for the bis(catecholato)diboron sample and protocol (2) was employed for the samples $\mathrm{Na}_{2} \mathrm{SO}_{4}$ and $\alpha-\mathrm{Al}_{2} \mathrm{O}_{3}$. The $2 \mathrm{Q}_{\mathrm{CT}}$ sideband manifold along the $\omega_{1}$ dimension will be depicted by a "stick-spectrum", where the height of each stick reflects the respective integrated sideband intensity, plotted against the sideband order $k$.

\subsection{Strategies for extracting inter-atomic distances and spin-cluster sizes}

2.2.1 Overview. As the number of spinning sidebands of significant amplitude in the CT 2Q NMR spectrum is proportional to the product $b_{j k} \tau_{\text {exc }}$, whenever an experimental result is available from one $\left\{b_{j k}, \tau_{\text {exc }}\right\}$ pair of parameters, a qualitative estimation of an unknown dipolar coupling constant is straightforward by counting the number of odd-order sidebands in the spectrum. For low values of $b_{j k} \tau_{\text {exc }}$, only the first-order $( \pm 1)$ sidebands appear, whereas the presence of a significant dipolar contact between two spins is signified by the growth of the higher-order $( \pm 3, \pm 5, \pm 7 \ldots)$ sidebands for increasing $2 \mathrm{Q}_{\mathrm{CT}}$ excitation intervals. ${ }^{46}$ Yet, accurate quantitative internucleardistance estimates must involve explicit numerical simulations of the spin dynamics in one way or the other. We then distinguish between two scenarios:

(A) The rare case when the internuclear separation within an isolated pair of half-integer spins is to be determined. Such spin systems may feature one internuclear distance $r_{j k}$ that is much shorter than all others; a typical scenario is a spin-pair within a molecular unit where the crystal structure involves an ordered arrangement of such units. This case may be handled by directly fitting the experimental data to numerically exact spin-pair simulations. ${ }^{46}$ Even for structures built from separate molecular units, truly isolated spin-pairs are extremely rare due to intermolecular dipolar interactions. This will be expanded on in Section 4.1, where we explore the consequences of including longer-range intermolecular couplings, and hence the deviation from a truly "isolated" ${ }^{11} \mathrm{~B}-{ }^{11} \mathrm{~B}$ pair, for determining of the $\mathrm{B}-\mathrm{B}$ interatomic distance in the bis(catecholato)diboron molecule.

In scenario (B) of a large continuous spin network, for which the CT 2Q sideband NMR experiment will create a significant number of recoupled spin-pairs, the size of such a spin- $I$ cluster grows for increasing $\tau_{\text {exc }}$. Then a large number of distinct spinpairs associated with a spread in $r_{j k}$-values contributes to the 
sideband pattern of the CT $2 \mathrm{Q}$ experiment. The measurement of a "single distance" is then not a well-defined concept for such cases, and one must simultaneously determine the large number of distances associated with the entire set of individual spin-pairs. Accurate numerical simulations cannot address this task due to the substantial time required to calculate the NMR spectrum from even a few dipolar coupled $I=3 / 2$ spins, and the prohibitive scaling of the computation time for higher-spin numbers. Numerically exact simulations accounting for more than four coupled spins-3/2 appears presently intractable by using standard computer resources, at least if employed for the purpose of iterative fitting to experimental data. Hence, one must resort to approximating the complex spin dynamics, as well as lower the ambition level from extracting multiple internuclear distances to just defining the spin cluster size. Here we employ the strategy of ref. 56, which amounts to first calculating the NMR spectrum from each unique spin-pair in the structure over given radius $R$-onwards denoted the "cut-off distance"followed by summation over all contributing spectra. Note that whereas each spin-pair is treated numerically exact, the procedure ignores all cross-couplings between spins of distinct pairs and their mutual interferences. Despite invoking a crude approximation that may not in general be justified rigorously, this strategy allowed for the successful determination of the absolute orientations of all ${ }^{23} \mathrm{Na}$ quadrupolar tensors in $\mathrm{Na}_{2} \mathrm{SO}_{3} ;{ }^{56}$ further validation of the approach is gained from the results presented in Section 4.1.

2.2.2 Spin-pair summation for calculating NMR spectra from multi-spin systems. Ref. 56 describes the numerical modeling procedure in detail; here we limit ourselves to recapitulating its key stages, as well as outlining the relationship between the number of dipolar interactions and number of contributing spins in the cluster.

For each pair $j k$ of unique crystallographic sites, all dipolar coupling constants and their accompanying orientations are extracted from the (known) crystal structure: firstly, the primitive unit cell is build from its corresponding asymmetric unit. Secondly, the central unit cell is extended into an odd order supercell, e.g. $3 \times 3 \times 3$ or $5 \times 5 \times 5$. Finally, all dipolar interactions from crystallographic sites in the central unit cell to sites in the same, as well as to surrounding, unit cells are located and sorted according to the relative absolute values of their dipolar coupling constants, i.e., by the corresponding internuclear distances. In the analysis below, dipolar interactions are considered up to the cut-off distance $R$. The corresponding minimum dipolar coupling constant is denoted by $b(R)=-\left(\mu_{0} / 4 \pi\right) \gamma_{I}^{2} \hbar R^{-3}$. Dipolar interactions that are related by translational symmetry are considered equivalent. Each of the inequivalent dipolar couplings is a member of one set of $M\left(b_{j k}\right)$ symmetry-related couplings, where $M\left(b_{j k}\right)$ is referred to as the dipolar multiplicity: ${ }^{56}$ all dipolar interactions within this set are associated with the same magnitude of $b_{j k}$, but differ in their orientations in the crystal structure. Concrete examples of this approach are provided in Sections 4.1-4.3 for the samples used in this study.

Next, the NMR spectrum for each dipolar vector is calculated: it may represent either the entire 2Q-1Q 2D correlation
NMR spectrum or its $\omega_{1}$-projection, depending on which underlying $t_{1}$-sampling approach is used (Section 2.1). This computation also involves all relevant chemical shift and quadrupolar interaction parameters, whose values must be a priori known. In general, all $M\left(b_{j k}\right)$ members that feature a fixed value of the dipolar coupling constant $b_{j k}$ may be divided into $N\left(b_{j k}\right)$ subsets. The NMR spectrum generated from each subset is distinct from that of any other subset, meaning that there are $N\left(b_{j k}\right)$ unique (i.e., distinct) spectra. In contrast, all dipolar coupling orientations within a given subset produce identical NMR spectra, owing to additional symmetries in the NMR responses. $^{38,56}$ Then explicit simulations must only be performed for one sole dipolar vector orientation from each of the $N\left(b_{j k}\right)$ subsets. The resulting set of dipolar coupling parameters is denoted $\left\{\left(b_{j k}, \Omega_{j k, i}^{\mathrm{DD}}\right)\right\}$, with the index $i$ running over all distinct subsets; $i=1, \ldots, N\left(b_{j k}\right)$. The corresponding individual simulated spectra are labeled $S_{j k}\left(\omega_{1}, \omega_{2} ; \tau_{\text {exc }}, b_{j k}, \Omega_{j k, i}^{\mathrm{DD}}\right)$. With this approach the computation time reduces by the factor $M\left(b_{j k}\right) / N\left(b_{j k}\right){ }^{56}$

The NMR spectrum $S_{\text {sum }}^{R}\left(\omega_{1}, \omega_{2} ; \tau_{\text {exc }}\right)$ that is representative of the entire spin system-the latter being defined by the $\tau_{\text {exc }^{-}}$ dictated radius $R$-is constructed by summing all individual contributions $S_{j k}\left(\omega_{1}, \omega_{2} ; \tau_{\text {exc }}, b_{j k}, \Omega_{j k, i}^{\mathrm{DD}}\right)$, while considering their respective dipolar multiplicities: ${ }^{56}$

$$
\begin{aligned}
S_{\text {sum }}^{R}\left(\omega_{1}, \omega_{2} ; \tau_{\text {exc }}\right)= & \sum_{j k}^{\text {unique sites }} \sum_{\left|b_{j k}\right| \geq|b(R)|} \frac{M\left(b_{j k}\right)}{N\left(b_{j k}\right)} \\
& \times \sum_{i=1}^{N\left(b_{j k}\right)} S_{j k}\left(\omega_{1}, \omega_{2} ; \tau_{\text {exc }}, b_{j k}, \Omega_{j k, i}^{\mathrm{DD}}\right) .
\end{aligned}
$$

The number $N_{\text {spins }}$ of interacting (re)coupled half-integer spins may be assessed by comparing the experimental $2 \mathrm{Q}_{\mathrm{CT}}$ sideband manifold with those calculated by eqn (1) for progressively expanded radii of the (potentially) infinitely large structure, with the aim of locating the $R$-value that minimizes the root mean square (RMS) deviation between experimental and calculated NMR spectra. Once the value of $R$ is determined, the resulting set of contributing dipolar interactions may be translated into the number of individual half-integer spins $N_{\text {spins }}$ involved in the corresponding coupling network, as follows: all distinct crystallographic sites in the constructed supercell are indexed consecutively. Next, the set of contributing dipolar interactions is translated into a set of index pairs, whereupon $N_{\text {spins }}$ is obtained from the number of unique spin indices found within this set.

\section{Materials and methods}

\subsection{Solid-state NMR experiments}

All experiments were performed at a static magnetic field of $B_{0}=11.75 \mathrm{~T}$ using a Bruker Avance-III spectrometer. The sample of bis(catecholato)diboron was studied at a ${ }^{11} \mathrm{~B}$ Larmor frequency $\left(-\gamma_{I} B_{0}\right)$ of $-160.5 \mathrm{MHz}$ in a Varian $3.2 \mathrm{~mm}$ doubleresonance standard-bore Varian MAS probehead. ${ }^{46}$ The powder of bis(catecholato)diboron was purchased from Sigma-Aldrich 
and was spun at $25 \mathrm{kHz}$. This corresponds to an rf field of $6.25 \mathrm{kHz}$ during dipolar recoupling, i.e., to a $\mathrm{CT}$ nutation frequency equal to half the MAS frequency, ${ }^{26}$ as for the HORROR condition..$^{20,21,74}$ The CT selective $90^{\circ}$ and $180^{\circ}$ pulses operated at $9.0 \mathrm{kHz}$ rf field amplitude. Complete 2D nutation experiments were performed to calibrate all rf amplitudes. To enhance the CT population difference, a single-frequency sweep (SFS) ${ }^{46}$ of $2 \mathrm{~ms}$ duration was performed at the same rf field-strength, by using an rf frequency offset and a sweep bandwidth of $500 \mathrm{kHz}$ and $800 \mathrm{kHz}$, respectively, where the beginning and end of the sweep-pulse shape were attenuated by a $\sin ^{2}$ and $\cos ^{2}$ function, respectively. The States-TPPI scheme was employed to obtain 2D pure absorption lineshapes and for distinguishing positive and negative $2 \mathrm{Q}$ coherences. ${ }^{75,76}$

The experiments on the $\mathrm{Na}_{2} \mathrm{SO}_{4}$ sample were performed on a $3.2 \mathrm{~mm}$ double-resonance standard-bore Varian MAS probehead at ${ }^{23} \mathrm{Na}$ Larmor frequency of $-132.3 \mathrm{MHz}$ and a spinning frequency of $20 \mathrm{kHz}$. The rf field during the $2 \mathrm{Q}_{\mathrm{CT}}$-recoupling was set to $5 \mathrm{kHz}$. The CT selective $90^{\circ}$ and $180^{\circ}$ pulses operated at $7.151 \mathrm{kHz}$. The CT population was enhanced by a SFS of $2 \mathrm{~ms}$ duration at the same rf field strength, $550 \mathrm{kHz}$ rf offset and $900 \mathrm{kHz}$ bandwidth, where the beginning and the end of the sweep where attenuated, as described above.

Prior to the NMR experiments, the sample of $\mathrm{Al}_{2} \mathrm{O}_{3}$ was heated to $1360{ }^{\circ} \mathrm{C}$ for $96 \mathrm{~h}$ to ensure a phase-pure $\alpha-\mathrm{Al}_{2} \mathrm{O}_{3}$ specimen, which was confirmed by a directly excited ${ }^{27} \mathrm{Al}$ MAS NMR spectrum. The NMR experiments were performed on a $4 \mathrm{~mm}$ double-resonance standard-bore Varian MAS probehead at a ${ }^{27} \mathrm{Al}$ Larmor frequency of $-130.3 \mathrm{MHz}$ and a spinning frequency of $10 \mathrm{kHz}$. The rf field during the $2 \mathrm{Q}_{\mathrm{CT}}$-recoupling was set to $1.674 \mathrm{kHz}$. The CT selective $90^{\circ}$ and $180^{\circ}$ pulses operated at $980 \mathrm{~Hz}$. The CT population was enhanced by a SFS of $2 \mathrm{~ms}$ duration at $2.577 \mathrm{kHz}$ rf field strength, $500 \mathrm{kHz}$ rf offset and $800 \mathrm{kHz}$ bandwidth, as described above.

\subsection{Numerical spin dynamics simulations}

The numerical simulations of the ${ }^{11} \mathrm{~B},{ }^{27} \mathrm{Al}$ and ${ }^{23} \mathrm{Na} 2 \mathrm{Q}_{\mathrm{CT}}$ sideband patterns were performed by using either the SIMPSON package (version 3.0.1) ${ }^{77}$ or software developed in Stockholm. ${ }^{27,41,56}$ The simulations invoked the experimental parameters listed in Section 3.1. All pulses were explicitly accounted for, except for the CT population enhancement and the $90^{\circ}$ read pulse.
Both the initial density operator and the detection operator represented CT $z$-magnetization. The CT 2QC were selected by nulling all other elements in the density matrix before and after the $180^{\circ}$ spin-echo pulse. The CT 2Q sideband amplitudes were simulated by incrementing $t_{1}$ in $N$ steps of duration $\tau_{\mathrm{r}} / N$, with $N$ equal to 35,35 and 16 for the simulations involving ${ }^{11} \mathrm{~B},{ }^{27} \mathrm{Al}$ and ${ }^{23} \mathrm{Na}$, respectively. The results of the separately calculated time-dependent signal amplitudes of the +2QC and $-2 \mathrm{QC}$ were combined to form "cosine" and "sine" NMR signal components required by the procedure of States et al. ${ }^{75}$ The resulting complex $t_{1}$-signal was subjected to a complex Fourier transformation to obtain the integrated CT 2Q spinning sideband amplitudes. Powder averaging was accomplished using a set of 6044 triplets of Euler angles selected according to the ZCW scheme. ${ }^{78-80}$ In all cases, we used the respective experimentally estimated values of the quadrupolar coupling constant and asymmetry parameter listed in Table 1. In case of bis(catecholato)diboron, the parameters of the CSA tensor and the orientation of the quadrupolar tensor as calculated by GIPAW-DFT were employed in the numerical simulations. Both for $\alpha-\mathrm{Al}_{2} \mathrm{O}_{3}$ and $\mathrm{Na}_{2} \mathrm{SO}_{4}$ the experimentally estimated value of the anisotropic chemical shift and asymmetry parameter were used together with the corresponding tensor orientations obtained by GIPAW-DFT calculations.

\subsection{Quantum chemical calculations}

For the GIPAW-DFT calculations of the quadrupolar and chemical shift tensors CASTEP ${ }^{81}$ and CASTEP-NMR ${ }^{82-84}$ (version 4.4) were used together with Accelrys' Materials Studio. The generalized gradient approximation with the Perdew-Burke-Ernzerhof exchange correlation functional ${ }^{85}$ and "on-the-fly" pseudopotentials were chosen. In all cases the plane wave cutoff energy was selected to be $550 \mathrm{eV}$. In case of bis(catecholato)diboron the $k$-point grid was set to $5 \times 2 \times 4$. Starting from the structure determined by single-crystal $\mathrm{XRD},{ }^{55}$ initially, only the proton positions were optimized, whereupon the NMR parameters were calculated. ${ }^{46}$ In case of $\mathrm{Na}_{2} \mathrm{SO}_{4}$ the conventional orthorhombic unit cell of the single-crystal structure $^{86}$ was converted to the primitive unit cell prior to the DFT calculations employing a $5 \times 5 \times 5 k$-point grid. For $\alpha-\mathrm{Al}_{2} \mathrm{O}_{3}$ the conventional hexagonal unit cell of the structure determined by single-crystal $\mathrm{XRD}^{87}$ was converted to the

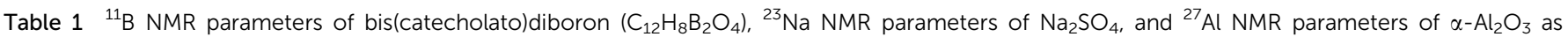

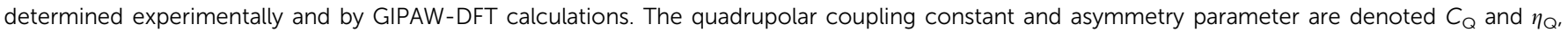

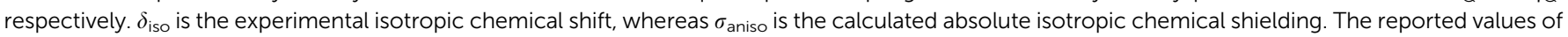

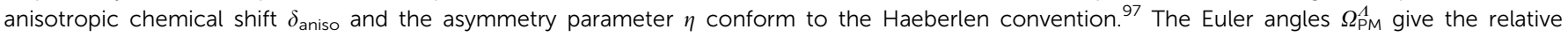
orientation of the principal axis system of the interaction tensor $\Lambda$ and a crystal fixed frame

\begin{tabular}{|c|c|c|c|c|c|c|c|c|c|c|c|c|c|c|}
\hline \multirow[b]{2}{*}{ Sample } & \multicolumn{6}{|c|}{ Experiment } & \multicolumn{8}{|c|}{ DFT calculations } \\
\hline & $\begin{array}{l}C_{\mathrm{Q}} \\
{[\mathrm{MHz}]}\end{array}$ & $\eta_{\mathrm{Q}}$ & $\begin{array}{l}\delta_{\text {iso }} \\
{[\mathrm{ppm}]}\end{array}$ & $\begin{array}{l}\delta_{\text {aniso }} \\
{[\mathrm{ppm}]}\end{array}$ & $\eta$ & Ref. & $\begin{array}{l}C_{\mathrm{Q}} \\
{[\mathrm{MHz}]}\end{array}$ & $\eta_{\mathrm{Q}}$ & $\begin{array}{l}\Omega_{\mathrm{PM}}^{\mathrm{Q}} \\
{\left[^{\circ}\right]}\end{array}$ & $\begin{array}{l}\sigma_{\text {iso }} \\
{[\mathrm{ppm}]}\end{array}$ & $\begin{array}{l}\delta_{\text {aniso }} \\
{[\mathrm{ppm}]}\end{array}$ & $\eta$ & $\begin{array}{l}\Omega_{\mathrm{PM}}^{\mathrm{CSA}} \\
{\left[{ }^{\circ}\right]}\end{array}$ & Ref. \\
\hline $\mathrm{C}_{12} \mathrm{H}_{8} \mathrm{~B}_{2} \mathrm{O}_{4}$ & 2.71 & 0.745 & 28.6 & - & - & 46 & 3.18 & 0.77 & $\{38.7,86.2,-135.0\}$ & -67.1 & -22.6 & 0.67 & $\{-38.9,95.0,49.1\}$ & 46 \\
\hline $\mathrm{Na}_{2} \mathrm{SO}_{4}$ & 2.6 & 0.6 & -8.5 & -18.9 & 0.0 & 37 and 98 & -3.20 & 0.60 & $\{-31.4,66.5,-166.4\}$ & -562.3 & -10.8 & 0.78 & $\{-61.5,86.1,-2.1\}$ & $\begin{array}{l}\text { This } \\
\text { work }\end{array}$ \\
\hline$\alpha-\mathrm{Al}_{2} \mathrm{O}_{3}$ & 2.40 & 0.0 & 18.8 & 17.3 & 0.0 & 99 & 2.29 & 0.0 & $\{0,0,0\}$ & 538.1 & 7.90 & 0.0 & $\{0,0,0\}$ & $\begin{array}{l}\text { This } \\
\text { work }\end{array}$ \\
\hline
\end{tabular}


primitive unit cell prior to the DFT calculations with a $6 \times 6 \times 6$ $k$-point grid.

\section{Results}

\subsection{The ${ }^{11} \mathrm{~B}-{ }^{11} \mathrm{~B}$ pair in bis(catecholato)diboron $(I=3 / 2)$}

We recently demonstrated that CT 2Q sideband NMR spectroscopy allowed for accurately determining the internuclear distance in the pair of directly bonded boron atoms in bis(catecholato)diboron by iterative fitting of numerically exact ${ }^{11} \mathrm{~B}-{ }^{11} \mathrm{~B}$ twospin simulations to experimental results, i.e., using approach (A) in Section 2.2.1. ${ }^{46}$

Nevertheless, despite a favorable accuracy and precision in the estimated distance, these two-spin simulations deviated systematically in their lowest-order $( \pm 1)$ sideband amplitudes relative to their experimental counterparts, despite that all higher-order sideband amplitudes agreed well. ${ }^{46}$ Here we analyze the source of these discrepancies further by employing numerically exact multi-spin simulations (comprising up to 4 spins) to account for the contributions from weaker intermolecular ${ }^{11} \mathrm{~B}-{ }^{11} \mathrm{~B}$ dipolar couplings between neighboring molecules, whose inclusion will be shown to provide excellent agreement with the experimental sideband patterns. Indeed, Holland et al. reported similar effects of intensified low-order sideband amplitudes in the context of ${ }^{1} \mathrm{H} \mathrm{NMR}$, and attributed them to the presence of distributions of dipolar-coupling strengths. ${ }^{88}$ We further demonstrate that the relatively effortless summation of sideband NMR spectra from a larger set of spin-pairs (i.e., strategy (B) in Section 2.2.1) reproduce the results from the rigorous multi-spin analysis. Noteworthy, the estimated internuclear distance of the directly bonded B-B pair remains largely unaffected by the approach for numerical analysis.

Fig. 2a shows the structure of a single bis(catecholato)diboron molecule together with the principal axis systems of the ${ }^{11} \mathrm{~B}$ chemical shift and quadrupolar tensors as obtained by GIPAWDFT calculations. ${ }^{46}$ Fig. $2 \mathrm{~b}$ displays the nearest neighboring molecules in the crystal structure, where the four boron sites in closest spatial contact are labeled by 1-4. From the XRD structure $^{55}$ the internuclear distances are determined to $r_{12}^{\mathrm{XRD}}=$ $168 \mathrm{pm}, r_{23}^{\mathrm{XRD}}=r_{14}^{\mathrm{XRD}}=382 \mathrm{pm}$ and $r_{13}^{\mathrm{XRD}}=r_{24}^{\mathrm{XRD}}=475 \mathrm{pm}$. The corresponding ${ }^{11} \mathrm{~B}-{ }^{11} \mathrm{~B}$ dipolar coupling constants are given by $b_{12}^{\mathrm{XRD}} / 2 \pi=-2619 \mathrm{~Hz}, b_{23}^{\mathrm{XRD}} / 2 \pi=-222 \mathrm{~Hz}$ and $b_{13}^{\mathrm{XRD}} / 2 \pi=-116 \mathrm{~Hz}$. Note that the intramolecular coupling constant of the directly bonded $(1,2)$ boron sites is $\approx 12$ times larger than the second largest intermolecular counterpart. In ref. 46 we therefore analyzed the experimental CT $2 \mathrm{Q}$ spinning sideband amplitudes by solely employing spin-pair simulations.

4.1.1 Spin-pair analysis. Fig. $3 \mathrm{~b}$ shows the integrated oddorder CT 2Q experimental ${ }^{11} \mathrm{~B}$ sideband amplitudes (dashed sticks) obtained from bis(catecholato)diboron for $\tau_{\text {exc }}=480 \mu \mathrm{s}$ at an external field of $11.75 \mathrm{~T}$ and spinning frequency of $25 \mathrm{kHz}$. The solid sticks depict the corresponding best-fit simulation for a ${ }^{11} \mathrm{~B}-{ }^{11} \mathrm{~B}$ spin pair with $b_{12} / 2 \pi=-2580 \mathrm{~Hz}$ (equivalent to $\left.r_{12}^{\mathrm{NMR}}=168.6 \mathrm{pm}\right)$. While the calculated intensities of all highorder sidebands agree very well with the experimental ones,
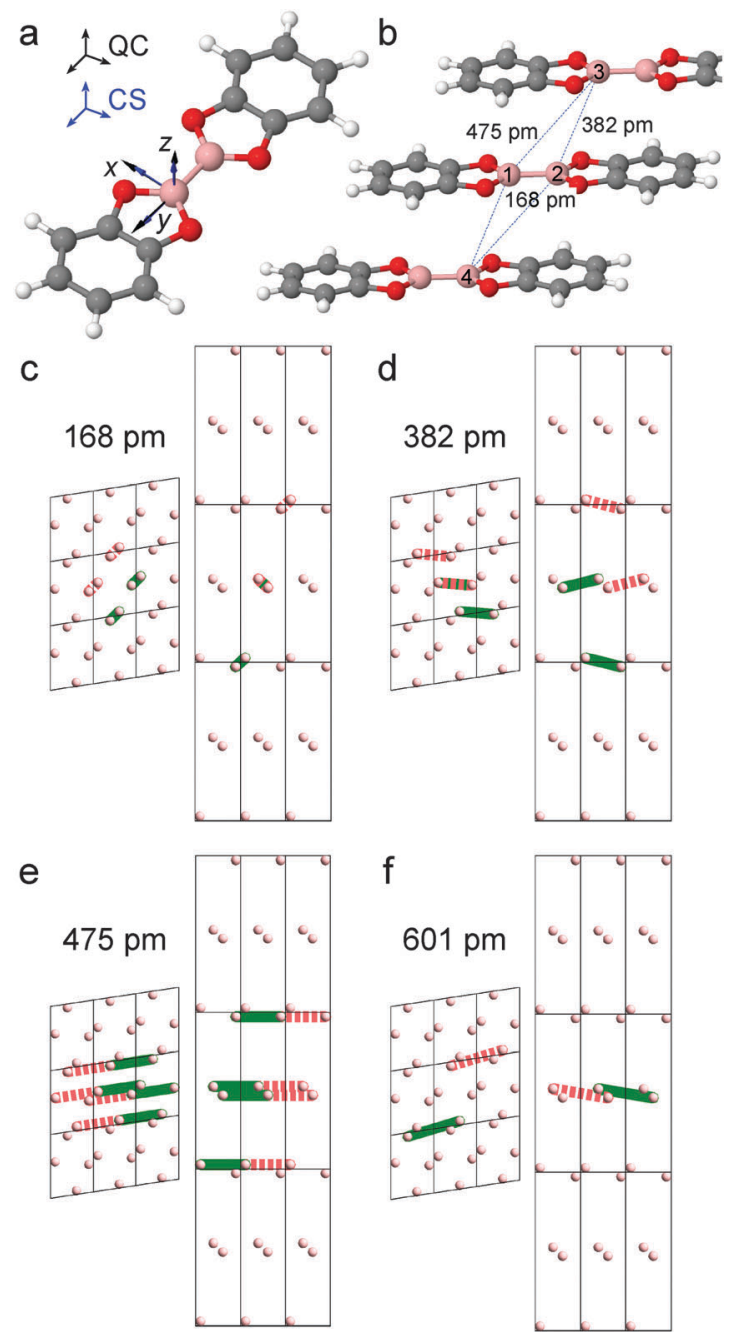

Fig. 2 (a) Molecular structure of bis(catecholato)diboron. ${ }^{55}$ The principal axis systems of the ${ }^{11} \mathrm{~B}$ quadrupole coupling $(\mathrm{QC})$ and chemical shift tensors (CS) as determined by CASTEP calculations are indicated. (b) Stacking of molecules in the bis(catecholato)diboron crystal structure. Selected intraand intermolecular $\mathrm{B}-\mathrm{B}$ distances are indicated, with the distinct boron sites labeled. (c)-(f) ${ }^{11} \mathrm{~B}-{ }^{11} \mathrm{~B}$ dipolar coupling network in the crystal structure of bis(catecholato)diboron. Contacts drawn with a solid line contribute to the multiplicity of a particular coupling, whereas dashed lines indicate equivalent couplings that do not contribute to the multiplicity. The individual ${ }^{11} \mathrm{~B}-{ }^{11} \mathrm{~B}$ dipolar coupling constants are given by (c) $-2619 \mathrm{~Hz}$, (d) $-222 \mathrm{~Hz}$, (e) $-116 \mathrm{~Hz}$, and (f) $-57 \mathrm{~Hz}$.

the experimental \pm 1 sidebands are more intense relative to those of the two-spin simulations. We performed a series of simulations by varying the dipolar coupling constant in steps of $10 \mathrm{~Hz}$ between -3080 and $-2080 \mathrm{~Hz}$. In each case, we calculated the sideband pattern and located the minimum of sum-squared deviation (SSD) between the simulated $\left(a_{\text {sim }}^{(k)}\right)$ and integrated experimental $\left(a_{\exp }^{(k)}\right)$ sideband amplitudes, only accounting for the sideband orders $k= \pm 3, \pm 5, \pm 7$, and \pm 9 :

$$
\mathrm{SSD}=\sum_{k}\left(a_{\mathrm{exp}}^{(k)}-A a_{\mathrm{sim}}^{(k)}\right)^{2}
$$

$A$ represents a free parameter that scales the simulated sideband amplitudes. 


\section{bis(catecholato)diboron}
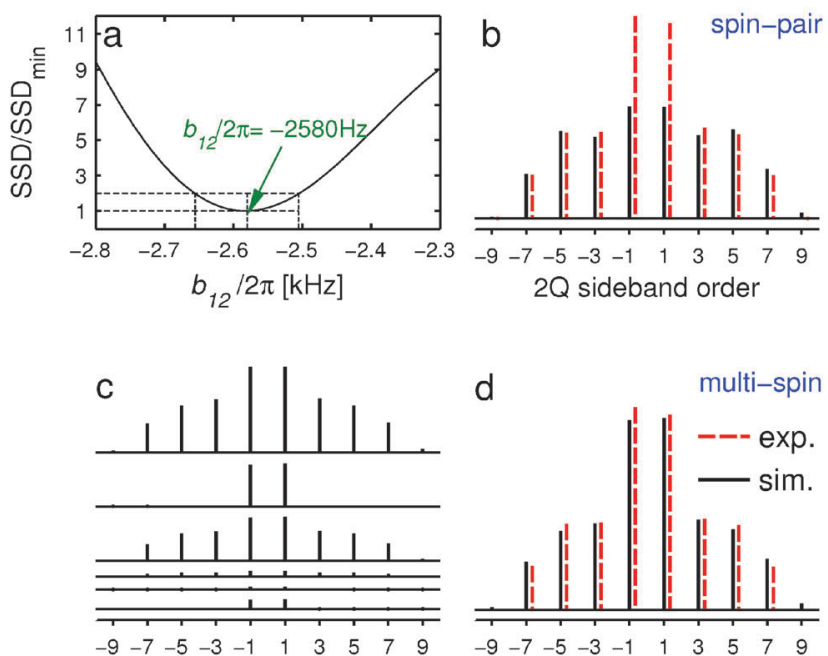

$2 \mathrm{Q}$ sideband order

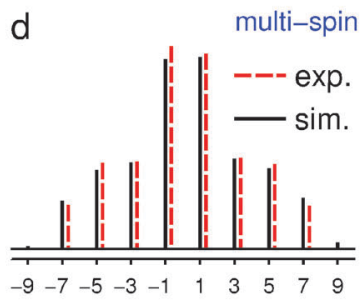

$2 \mathrm{Q}$ sideband order
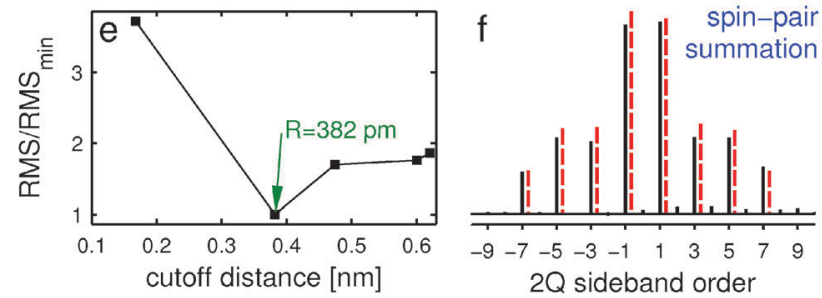

Fig. 3 The three rows show the result of using the spin-pair, multi-spin and spin-pair summation approaches to analyze the experimental ${ }^{11} \mathrm{~B} C \mathrm{CT}$ $2 \mathrm{Q}$ spinning sideband amplitudes obtained in bis(catecholato)diboron for $\tau_{\text {exc }}=480 \mu \mathrm{s}$. Experimental and simulated $\mathrm{CT} 2 \mathrm{Q}$ spinning sideband amplitudes are depicted for comparison as dashed as solid sticks, respectively, in the right column. (a) Normalized sum-squared deviation (SSD) of experimental and spin-pair simulated CT 2Q spinning sideband amplitudes against the ${ }^{11} \mathrm{~B}-{ }^{11} \mathrm{~B}$ dipolar coupling constant. The $95 \%$ confidence interval is indicated. (b) The best-fit numerical spin-pair simulations depicted as solid lines are for $\mathrm{a}^{11} \mathrm{~B}-{ }^{11} \mathrm{~B}$ dipolar coupling constant of $b_{12} / 2 \pi=-2580 \mathrm{~Hz}$. (c) Individual multi-spin simulated CT $2 \mathrm{Q}$ spinning sideband amplitude patterns for the different ${ }^{11} \mathrm{~B}$ multiple spin-systems shown in Fig. 2. The spin systems are given with their respective contribution from bottom to top by: $5.1 \%(4-1 / 2-3) ; 5.1 \%(4-2 / 1-3) ; 2.5 \%(1-2) ; 20.5 \%(4-1-2 / 1-2-3)$; $20.5 \%(4-1-3 / 4-2-3) ; 41.2 \%(4-1-2-3)$. (d) The simulations shown as solid lines correspond to the sum of multi-spin contributions in (c). (e) Normalized root-mean-square (RMS) deviation between experimental CT $2 Q$ spinning sideband amplitudes and summed spin-pair simulations plotted against the cut-off distance $R$. (f) The solid sticks represent the sum of numerical simulations for $\mathrm{B}-\mathrm{B}$ distances out to $R=381.9 \mathrm{pm}$, corresponding to the minimum of the RMS deviation in (e).

Fig. 3a plots the ratio $\mathrm{SSD} / \mathrm{SSD}_{\min }$ against the dipolar coupling constant $b_{12} / 2 \pi$, where $\mathrm{SSD}_{\min }$ represents the deviation obtained for the best fit. The $95 \%$ confidence interval, indicated in the figure, was determined by the set of dipolar couplings that obey $\mathrm{SSD} \leq \operatorname{SSD}_{\min }\left\{1+F_{1,6}^{0.05} / 6\right\}$, where $F^{\alpha}\left(p_{1}, p_{2}\right)$ is the upper $\alpha$ probability point of the $F$ distribution with $p_{1}$ and $p_{2}$ degrees of freedom. ${ }^{89,90}$ The resulting $95 \%$ confidence interval for the ${ }^{11} \mathrm{~B}-{ }^{11} \mathrm{~B}$ dipolar coupling constant is $-2580 \pm 75 \mathrm{~Hz}$, corresponding to a solid-state NMR determined distance of $168.6(+1.7,-1.6) \mathrm{pm}$. In ref. 46 , we also showed that varying the ${ }^{11} \mathrm{~B}$ quadrupolar coupling constant in the range of $(-3.5,-2.1) \mathrm{MHz}$ had a very weak effect on the sideband amplitudes. This generally also applies to the orientations of the quadrupolar interaction, as well as to the magnitude and orientation of the CSA tensor. After accounting for the potential contributions from these interactions, our analysis amounted in a reliable estimate of the ${ }^{11} \mathrm{~B}-{ }^{11} \mathrm{~B}$ dipolar coupling constant as $-2580(+140,-120) \mathrm{Hz}$, i.e., corresponding to a $\mathrm{B}-\mathrm{B}$ distance of $(168.6 \pm 3) \mathrm{pm}$, with relative uncertainties of about $\pm 5 \%$ and $\pm 2 \%$ in the dipolar coupling constant and the internuclear distance, respectively.

The observed dipolar coupling constant is about $1.5 \%$ smaller than that calculated from the XRD structure, and the corresponding NMR determined distance is only about $0.5 \%$ larger than that obtained from XRD. This small discrepancy can be attributed to the thermal motion of the boron positions: for the case of glycine, Ishii et al. calculated by molecular dynamics simulations that direct bond distances between non-proton nuclei measured by NMR at $273 \mathrm{~K}$ are typically $1.9-2.9 \%$ longer than the distances estimated by diffraction techniques. ${ }^{91}$ Furthermore, the anisotropy of the $J$-coupling $(\Delta J)$ has the same symmetry under sample rotation and rf pulses as the direct dipolar interaction. Hence, in principle, only the effective spin-spin interaction $D_{\text {eff }}=b_{12} / 2 \pi-\Delta J / 3$ can be determined with the CT 2Q sideband experiment. ${ }^{92}$ However, DFT calculations (not shown here), indicate that $\Delta J$ is in the order of 13-17 $\mathrm{Hz}$ for the ${ }^{11} \mathrm{~B}-{ }^{11} \mathrm{~B}$ pair in bis-catecholato-diboron. Consequently, its potential bearings on the estimation of the dipolar coupling is negligible compared to the effects from thermal motions.

4.1.2 Multi-spin analysis. Owing to the high natural abundance of $80.1 \%$ for ${ }^{11} \mathrm{~B}$ and the close molecular packing in the crystal structure of bis(catecholato)diboron (see Fig. 2b), longrange ${ }^{11} \mathrm{~B}-{ }^{11} \mathrm{~B}$ dipolar interactions need to be considered for explaining the intense $\pm 1 \mathrm{CT} 2 \mathrm{Q}$ spinning sidebands. Table S1 of the ESI $\dagger$ summarizes the various probabilities of finding a certain multiple- ${ }^{11} \mathrm{~B}$ spin system for these 4 boron sites, revealing that isolated ${ }^{11} \mathrm{~B}^{-11} \mathrm{~B}$ spin pairs are rarely encountered in the structure. While the remaining $19.9 \%$ boron isotopes are spin-3 ${ }^{10} \mathrm{~B}$ nuclei, the heteronuclear ${ }^{11} \mathrm{~B}-{ }^{10} \mathrm{~B}$ dipolar interactions are effectively decoupled during the $2 \mathrm{Q}_{\mathrm{CT}}$-recoupling sequence on the ${ }^{11} \mathrm{~B}$.

Fig. 3c shows the calculated CT 2Q sideband amplitude patterns obtained from the various ${ }^{11} \mathrm{~B}$ multiple-spin systems by employing numerically exact simulations; each is scaled by the corresponding probability of finding that particular constellation of coupled ${ }^{11} \mathrm{~B}$ sites (see Table $\mathrm{S} 1$ in the ESI $\dagger$ ). Fig. 3d contrasts their sum (solid sticks) with the experimental amplitudes (dashed sticks): an excellent agreement is observed. We stress that the present summation procedure is only required due to the presence of the ${ }^{10} \mathrm{~B}$ isotope in the sample; it must not be confused with the analogous spin-pair summation protocol described in Section 2.2.2 and utilized in Section 4.1.3, as well as for the $100 \%$ abundant ${ }^{27} \mathrm{Al}$ and ${ }^{23} \mathrm{Na}$ nuclides below.

4.1.3 Spin-pair summation. We further applied approach (B) of Section 2.2.2 that derives the NMR spectrum by summation over all NMR responses from the individual spin-pairs 
encountered within a given cut-off distance $R$. Fig. $2 \mathrm{c}-\mathrm{f}$ show the boron sites in the crystal structure of bis(catecholato)diboron. Each reveals the different ${ }^{11} \mathrm{~B}-{ }^{11} \mathrm{~B}$ site pairs with a particular internuclear distance and dipolar coupling. Site pairs that contribute to the multiplicity $M_{j k}$ of the respective coupling are depicted as solid lines, whilst equivalent pairs not contributing to that particular multiplicity are drawn with dashed lines. Table S2 in the ESI $\dagger$ summarizes all distinct ${ }^{11} \mathrm{~B}-{ }^{11} \mathrm{~B}$ sitepair and their corresponding multiplicities up to $R=621 \mathrm{pm}$, as well as the orientation of each dipolar vector with respect to the crystal frame.

Fig. 3e shows the RMS deviation between the experimental $2 \mathrm{Q}_{\mathrm{CT}}$-sideband amplitudes and the sum of two-spin simulations according to eqn (1), plotted against $R$. The RMS calculation accounted for all odd sideband orders $k= \pm 1, \pm 3, \pm 5$, $\pm 7, \pm 9$, with each RMS value normalized with respect to its minimum $\left(\mathrm{RMS}_{\min }\right)$ found at $R=382 \mathrm{pm}$; Fig. 3 f contrasts the respective sideband pattern obtained by summing all two-spin simulations (solid sticks) with the corresponding experimental result (dashed sticks). The very good agreement observed between the simulation and experiment is only marginally worse than that by using numerically exact multi-spin simulations in Fig. 3d. This validates the approach leading to eqn (1), i.e. that the spin dynamics of a large number of mutually dipolar-coupled spins may in these applications be reasonably well approximated by a sum over pair-wise responses.

\subsection{The ${ }^{23} \mathrm{Na}(I=3 / 2)$ network in $\mathrm{Na}_{2} \mathrm{SO}_{4}$}

We next consider application of CT 2Q sideband NMR spectroscopy to the dipolar-coupled network of ${ }^{23} \mathrm{Na}$ spins $(I=3 / 2)$ in $\mathrm{Na}_{2} \mathrm{SO}_{4}$, which involves one crystallographically unique $\mathrm{Na}$ site. Fig. 4a illustrates the single-crystal XRD-derived structure, ${ }^{86}$ also indicating the directions of the principal axes of the ${ }^{23} \mathrm{Na}$ chemical shift and quadrupolar tensors, as obtained by GIPAWDFT calculations. Fig. 4b-e convey the spatial relationship between the various equivalent and inequivalent ${ }^{23} \mathrm{Na}-{ }^{23} \mathrm{Na}$ pairs. Table S2 in the ESI $\dagger$ summarizes all inequivalent ${ }^{23} \mathrm{Na}-{ }^{23} \mathrm{Na}$ dipolar interactions, the corresponding multiplicities, and vector directions over a cut-off radius of $R=590 \mathrm{pm}$.

Fig. 5 plots the experimental ${ }^{23} \mathrm{Na} 2 \mathrm{Q}_{\mathrm{CT}}$-filtered $\left(2 \mathrm{QF}_{\mathrm{CT}}\right)$ efficiencies against the excitation interval $\tau_{\text {exc }}$. The results were obtained at a spinning frequency of $20 \mathrm{kHz}$, an external field of 11.75 $\mathrm{T}$ and two distinct $\mathrm{rf}$ carrier frequency positions (relative to the center of mass of the ${ }^{23} \mathrm{Na}$ powder lineshape); this produced resonance offsets of $0 \mathrm{~Hz}$ and $1080 \mathrm{~Hz}$, and the associated maximum $2 \mathrm{QF}_{\mathrm{CT}}$ efficiencies of $15.9 \%$ and $23.4 \%$, respectively. However, regardless of the precise value of the resonance offset, an optimum signal intensity was observed at $\tau_{\text {exc }}=\tau_{\text {exc }}^{\max }=0.8 \mathrm{~ms}$.

The left panel of Fig. 6 shows RMS deviations between experimental 2Q $\mathrm{Q}_{\mathrm{CT}}$-sideband amplitudes and those calculated by summing over simulated two-spin responses [eqn (1)], plotted against the cut-off distance $R$, and with $2 \mathrm{Q}_{\mathrm{CT}}$ excitation periods increasing from top to bottom; each curve is normalized with respect to its respective minimum. The RMS deviations were determined both by including (squares) and omitting
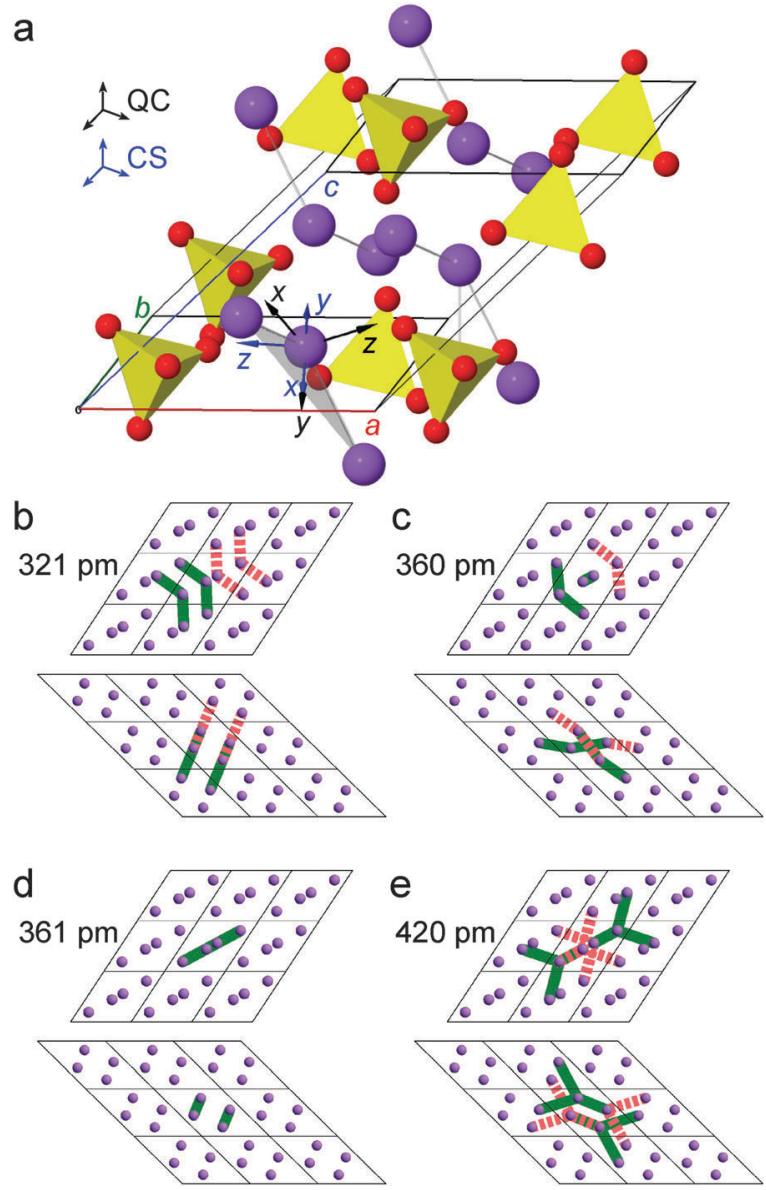

Fig. 4 (a) Crystal structure of $\mathrm{Na}_{2} \mathrm{SO}_{4}$ determined by XRD. ${ }^{86}$ The principal axis systems of the ${ }^{23} \mathrm{Na}$ quadrupole coupling (QC) and chemical shift (CS) tensors as determined by CASTEP calculations are indicated. (b) $-(e){ }^{23} \mathrm{Na}-{ }^{23} \mathrm{Na}$ dipolar coupling network in the crystal structure of $\mathrm{Na}_{2} \mathrm{SO}_{4}$. Contacts depicted as solid lines contribute to the multiplicity of a particular coupling, whereas contacts shown by dashed lines are equivalent couplings that do not contribute to the multiplicity. The individual ${ }^{23} \mathrm{Na}-{ }^{23} \mathrm{Na}$ dipolar coupling constants are given by (b) $-254 \mathrm{~Hz}$, (c) $-181 \mathrm{~Hz}$, (d) $-180 \mathrm{~Hz}$, and (e) $-113.5 \mathrm{~Hz}$.

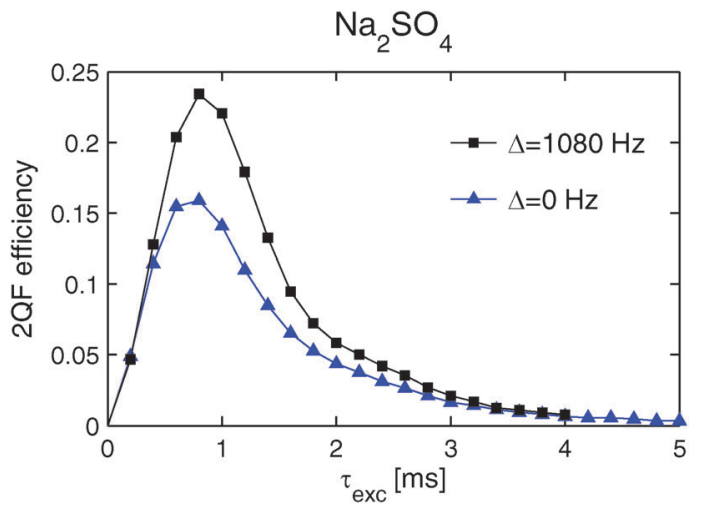

Fig. 5 Experimental ${ }^{23} \mathrm{Na} 2 \mathrm{Q}_{\mathrm{CT}}$-filtered efficiencies plotted against $\tau_{\text {exc. }}$. They were obtained from $\mathrm{Na}_{2} \mathrm{SO}_{4}$ by using the pulse sequence shown in Fig. 1 at a spinning frequency of $20 \mathrm{kHz}$, an external field of $11.75 \mathrm{~T}$, and two distinct rf offsets: $\Delta 0 \mathrm{~Hz}$ and $\mathbf{\square} 1080 \mathrm{~Hz}$. 


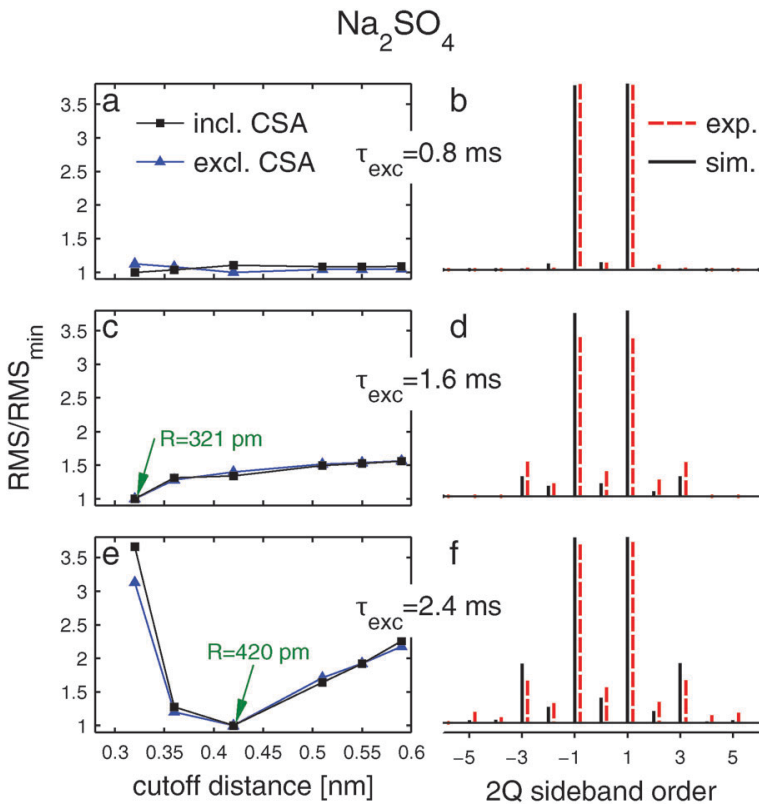

Fig. 6 Left column: normalized RMS deviations between experimental ${ }^{23} \mathrm{Na} \mathrm{CT} 2 \mathrm{Q}$ spinning sideband amplitudes and summed spin-pair simulations in $\mathrm{Na}_{2} \mathrm{SO}_{4}$ plotted against the maximum $\mathrm{Na}-\mathrm{Na}$ internuclear distance included in the summation. $\boldsymbol{\Delta}$ and $\boldsymbol{\nabla}$ correspond to the results obtained in the absence and presence of the CSA in the simulations, respectively. Right column: dashed sticks depict the experimental CT $2 Q$ sideband amplitudes. Solid sticks represent the sum of numerical simulations obtained by including all $\mathrm{Na}-\mathrm{Na}$ distances up to the $R$-value marked in the left column.

(triangles) CSA in the calculations. The right panel contrasts the experimental CT $2 \mathrm{Q}$ sideband amplitude manifold (dashed sticks) with the best-fit simulation incorporating CSA (solid sticks).

At the shortest $2 \mathrm{Q}_{\mathrm{CT}}$ excitation interval of $\tau_{\mathrm{exc}}=0.8 \mathrm{~ms}$, solely the lowest order $( \pm 1)$ sidebands are present in the manifold (Fig. 6b) that depends marginally on the cut-off distance (Fig. 6a); this feature precludes the probing of long-range $\mathrm{Na}-\mathrm{Na}$ internuclear distances. The middle row of Fig. 6 displays the results for $\tau_{\mathrm{exc}}=1.6 \mathrm{~ms}$. The experimental $2 \mathrm{Q}_{\mathrm{CT}}$-sideband pattern now comprises sidebands up to orders \pm 3 and the RMS deviation varies significantly when $R$ changes; it minimizes at $R=321 \mathrm{pm}$, regardless of whether CSA was accounted for in the simulations. The latter applies throughout all cases examined in the present work. The results of lengthening the excitation period further $\left(\tau_{\text {exc }}=2.4 \mathrm{~ms}\right)$ are shown in Fig. $6 \mathrm{e}$ and $\mathrm{f}$ that reveal $2 \mathrm{Q}_{\mathrm{CT}}$-sideband patterns incorporating orders \pm 5 and also the most pronounced sensitivity of the RMS deviation when the cut-off distance alters. As expected, the best-fit simulation is obtained at an expanded radius $(R=420 \mathrm{pm})$ relative to that at the shorter $\tau_{\text {exc }}$ values. The simulated and experimental $2 \mathrm{Q}_{\mathrm{CT}}$-sideband patterns agree very well. Hence, this choice of $2 \mathrm{Q}_{\mathrm{CT}}$ excitation time is preferable to probe longerrange structural fragments in the ${ }^{23} \mathrm{Na}$ nuclear spin network of $\mathrm{Na}_{2} \mathrm{SO}_{4}$.

As opposed to the confined ${ }^{11} \mathrm{~B}$ spin system in bis(catecholato)diboron, the macroscopic network of ${ }^{23} \mathrm{Na}$ spins in $\mathrm{Na}_{2} \mathrm{SO}_{4}$ precludes the determination of individual internuclear distances.
Table 2 The number of spin-pairs ( $\left.N_{\text {pairs }}\right)$ and corresponding number of unique spins ( $N_{\text {spins }}$ ) that are part of the dipolar coupling network at the cut-off distance $R$ corresponding to the minimum in the RMS curve of experimental and simulated sideband manifolds for the different samples and $2 Q_{\mathrm{CT}}$ excitation times $\tau_{\text {exc }}$ used

\begin{tabular}{lllcc}
\hline Sample & $\tau_{\text {exc }}[\mathrm{ms}]$ & $R[\mathrm{pm}]$ & $N_{\text {pairs }}$ & $N_{\text {spins }}$ \\
\hline $\mathrm{C}_{12} \mathrm{H}_{8} \mathrm{~B}_{2} \mathrm{O}_{4}$ & 0.48 & 382 & 8 & 12 \\
$\mathrm{Na}_{2} \mathrm{SO}_{4}$ & 1.6 & 321 & 8 & 12 \\
& 2.4 & 420 & 31 & 22 \\
$\alpha-\mathrm{Al}_{2} \mathrm{O}_{3}$ & 0.4 & 266 & 2 & 4 \\
& 0.8 & 279 & 14 & 16 \\
& 1.0 & 350 & 50 & 34
\end{tabular}

Structural analyses must rather aim at validating if the atomic coordinates (and its associated set of dipolar interactions) of a proposed structural model may reproduce the experimental results. At a selected $2 \mathrm{Q}_{\mathrm{CT}}$ excitation interval, the experiment provides the number $N_{\text {spins }}$ of interacting spins within the macroscopically large spin ensemble. For a finite-sized cluster in a structure, $N_{\text {spins }}$ corresponds to its number of spins. The value of $N_{\text {spins }}$ may then be estimated by locating the $R$-value that minimizes the RMS deviation between the experimental $2 \mathrm{Q}_{\mathrm{CT}}$ sideband manifold and the sum over all simulated spinpair responses therein. Table 2 lists the cluster-size ( $\left.N_{\text {spins }}\right)$ of the $\mathrm{Na}_{2} \mathrm{SO}_{4}$ structure assessed at each excitation period; $N_{\text {spins }}$ increases from 12 to 22 when $\tau_{\text {exc }}$ increases from $1.6 \mathrm{~ms}$ to $2.4 \mathrm{~ms}$.

We note that Duer $^{37}$ used a related approach to analyze the ${ }^{23} \mathrm{Na}^{2}{ }^{23} \mathrm{Na}$ dipolar coupling-network in $\mathrm{Na}_{2} \mathrm{SO}_{4}$, by modeling the experimental triple-quantum MAS (3QMAS) ${ }^{93}$ NMR spectrum via numerical simulations that also accounted for multiple homonuclear couplings. This experiment produces a sideband manifold stemming from the rotor-modulated evolution of (single-spin) 3QC under the, by slow-MAS incompletely averaged, homonuclear ${ }^{23} \mathrm{Na}^{23} \mathrm{Na}$ couplings, which in the absence of explicit homonuclear dipolar-recoupling were truncated to solely comprise the mutually commuting $I_{j z} I_{k z}$ operators of the dipolar Hamiltonians in the analysis. ${ }^{37}$ By including the homonuclear couplings to the five nearest neighbors, Duer successfully reproduced the isotropic projection of the 3QMAS experiment by exploiting the known dipolar coupling-constants and orientations in the crystal structure of $\mathrm{Na}_{2} \mathrm{SO}_{4}$. This approach differs to ours, both concerning the origin of the sideband-formation, as well as in the numerical analysis, where we include the full form of the (high-field) dipolar Hamiltonian, yet assuming a set of mutually non-interacting spin-pairs.

\subsection{The ${ }^{27} \mathrm{Al}(I=5 / 2)$ network in $\alpha-\mathrm{Al}_{2} \mathrm{O}_{3}$}

We further evaluated the CT $2 \mathrm{Q}$ sideband NMR experimentation and its accompanying "spin-counting" analysis in the context of the ${ }^{27} \mathrm{Al}$ spins $(I=5 / 2)$ in $\alpha-\mathrm{Al}_{2} \mathrm{O}_{3}$, which involves one crystallographically unique $\mathrm{Al}$ site coordinated by oxygen atoms. Fig. 7a reveals a very densely populated network of $\mathrm{Al}$, stemming from the presence of face-shared $\mathrm{AlO}_{6}$ octahedra. The GIPAW-DFT-derived principal axis systems of the ${ }^{27} \mathrm{Al}$ chemical shift and quadrupolar tensors are shown in Fig. $7 a$ and b. 
a

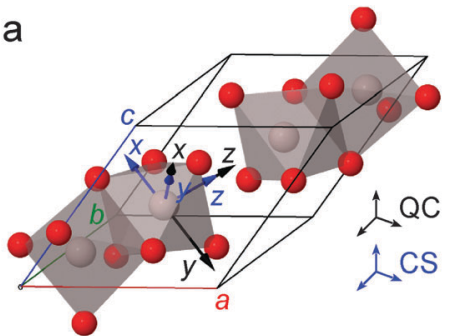

b

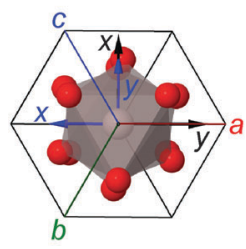

C

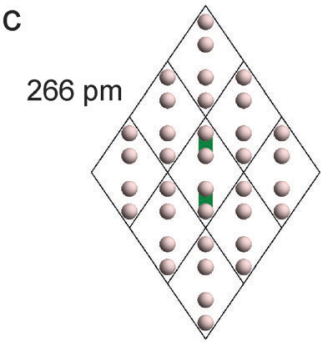

e

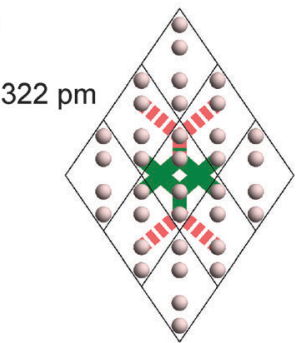

d

$279 \mathrm{pm}$

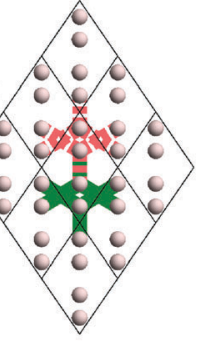

$f$

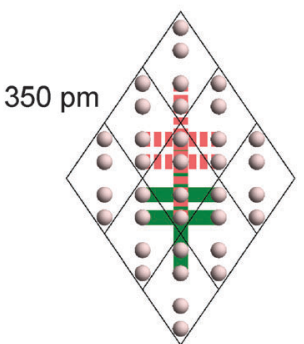

Fig. 7 (a, b) Crystal structure of $\alpha-\mathrm{Al}_{2} \mathrm{O}_{3}$ determined by XRD. ${ }^{87}$ The principal axis systems of the ${ }^{27} \mathrm{Al}$ quadrupole coupling and chemical shift tensors as determined by CASTEP calculations are indicated. (c) $-(\mathrm{f}){ }^{27} \mathrm{Al}-{ }^{27} \mathrm{Al}$ dipolar coupling network in the crystal structure of $\alpha-\mathrm{Al}_{2} \mathrm{O}_{3}$. The line styles are explained in the caption of Fig. 4. The individual ${ }^{27} \mathrm{Al}-{ }^{27} \mathrm{Al}$ dipolar coupling constants are given by (c) $-436 \mathrm{~Hz}$, (d) $-375 \mathrm{~Hz}$, (e) $-245 \mathrm{~Hz}$, and (f) $-191 \mathrm{~Hz}$.

A selection of inequivalent $\mathrm{Al}-\mathrm{Al}$ pairs is shown in Fig. $7 \mathrm{c}-\mathrm{f}$, while all coupling constants, multiplicities and dipolar vector orientations out to $R=591 \mathrm{pm}$ are listed in Table $\mathrm{S} 2$ in the ESI. $\dagger$

Fig. 8 plots experimental ${ }^{27} \mathrm{Al} 2 \mathrm{QF}_{\mathrm{CT}}$ efficiencies against increasing $\tau_{\text {exc }}$-values, as obtained from $\alpha-\mathrm{Al}_{2} \mathrm{O}_{3}$ at a magnetic field of $11.75 \mathrm{~T}$ and $10 \mathrm{kHz}$ MAS frequency. A maximum $2 \mathrm{QF}_{\mathrm{CT}}$

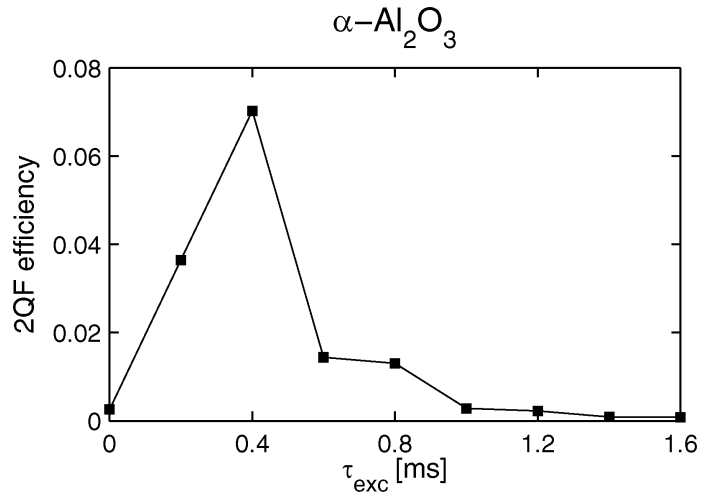

Fig. 8 Experimental ${ }^{27} \mathrm{Al} 2 \mathrm{Q}_{\mathrm{CT}}$-filtered efficiencies obtained from $\alpha-\mathrm{Al}_{2} \mathrm{O}_{3}$ and plotted against $\tau_{\text {exc }}$. The pulse sequence shown in Fig. 1 was employed at a spinning frequency of $10 \mathrm{kHz}$ and an external field of $11.75 \mathrm{~T}$.

efficiency of $7 \%$ was obtained around $\tau_{\text {exc }}=0.4 \mathrm{~ms}$, which is considerably lower than the ${ }^{23} \mathrm{Na} 2 \mathrm{QF}_{\mathrm{CT}}$ efficiency in $\mathrm{Na}_{2} \mathrm{SO}_{4}$. While these signal losses stem partially from a more rapid ${ }^{27} \mathrm{Al}$ relaxation during $\mathrm{rf}$ application compared to that for ${ }^{23} \mathrm{Na}$ in $\mathrm{Na}_{2} \mathrm{SO}_{4}$, they primarily originate from a significantly higher CT-magnetization leakage out to the satellite transitions for a spin-5/2 compared to the spin-3/2 case, as discussed in ref. 15 and 28. Furthermore, for a fixed dipolar-coupling constant, the 2QC buildup rate is higher for a $I=5 / 2$ pair compared to that of two spins-3/2 (vide infra); ${ }^{28}$ together with the overall stronger ${ }^{27} \mathrm{Al}-{ }^{27} \mathrm{Al}$ dipolar coupling constants in the $\alpha-\mathrm{Al}_{2} \mathrm{O}_{3}$ structure (see Table S2, ESI $\dagger$ ), this accounts for the nearly doubled 2QC generation rate; compare the results of Fig. 5 and 8.

Fig. 9 displays the experimental and simulated $2 \mathrm{Q}_{\mathrm{CT}}$-sideband spectra for increasing $2 \mathrm{Q}_{\mathrm{CT}}$ excitation periods. At the shortest value $\tau_{\text {exc }}=\tau_{\text {exc }}^{\max }=0.4 \mathrm{~ms}$ where the $2 \mathrm{QF}_{\mathrm{CT}}$ efficiency is optimized (Fig. 9a and b), the experimental 2Q $\mathrm{Q}_{\mathrm{CT}}$-sideband spectrum reveals only the \pm 1 sideband orders. The best-fit spin-pair sum was obtained from the set of couplings over $R=266 \mathrm{pm}$, corresponding to only two $\mathrm{Al}-\mathrm{Al}$ pairs and a cluster of four ${ }^{27} \mathrm{Al}$ sites (i.e., $N_{\text {spins }}=4$ ); see Table 2 . On the other hand, at $\tau_{\text {exc }}=0.8 \mathrm{~ms}$ (Fig. 9c), the sideband manifold comprises both \pm 1 and \pm 3 sideband orders. Now only a fair agreement is observed for the "best-fit" NMR sideband pattern, which resulted by summing the simulated responses from all pairs over $R=279 \mathrm{pm}$ $\left(N_{\text {pairs }}=14\right)$, and translating into a cluster comprising 16 unique ${ }^{27} \mathrm{Al}$ sites. At the longest $2 \mathrm{Q}_{\mathrm{CT}}$ excitation period $\tau_{\mathrm{exc}}=1.0 \mathrm{~ms}$ (Fig. 9f), the experimental $2 \mathrm{Q}_{\mathrm{CT}}$-sideband pattern includes all

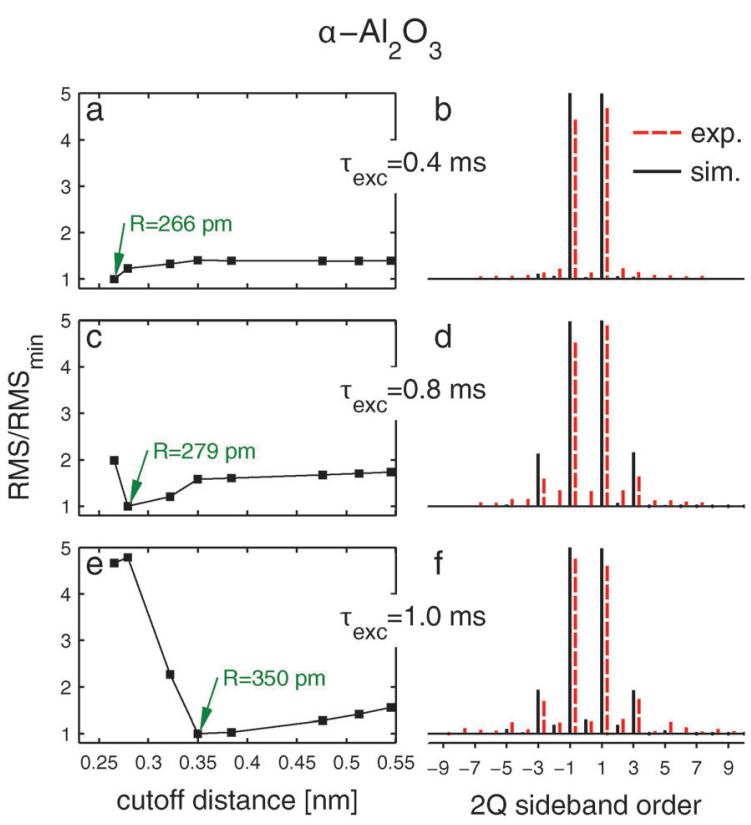

Fig. 9 Left column: normalized RMS deviations between the results from experimental $2 Q_{C T}$ and summed spin-pair simulated spinning sideband amplitudes from $\alpha-\mathrm{Al}_{2} \mathrm{O}_{3}$, and displayed for increasing cut-off distance $R$ used in the spin-pair summation. Right column: dashed sticks depict the experimental CT $2 Q$ sideband amplitudes. Solid sticks represent the sum of numerical simulations resulting by including all Al-Al distances up to the $R$-value indicated in the left column. 
sideband orders up to \pm 5 , although the amplitudes of the highest order are very weak. The experimental result is wellreproduced by a sum over 50 spin-pairs within $R=350 \mathrm{pm}$, corresponding to a cluster comprising 34 spins (Table 2).

\section{Discussion}

Here we discuss the prospects of applying each of strategies (A) and (B) of Section 2.2 for analyzing dipolar-coupled spin networks and estimating internuclear distances.

\subsection{Estimating internuclear distances}

The results for the ${ }^{11} \mathrm{~B}$ spin-3/2 pair in bis(catecholato)diboron demonstrates that CT $2 \mathrm{Q}$ spectroscopy is an excellent method to accurately determine internuclear distances within (isolated) pairs of half-integer quadrupolar nuclei. The sensitivity of the method increases with the number of $2 \mathrm{Q}_{\mathrm{CT}}$ sideband orders that are considered in the analysis. Given that the number of spinning sidebands with significant intensity depends on the product $b_{j k} \tau_{\text {exc }}$, the excitation period may be progressively increased to ensure that a sufficiently large sideband manifold is available for the estimation of weak dipolar coupling constants, whereas the upper limit of the $\tau_{\text {exc }}$ is only limited by signaldamping effects from relaxation. For a truly isolated spin-pair, the dipolar coupling constant is then readily extracted through effortless two-spin simulations: the calculated $2 \mathrm{Q}_{\mathrm{CT}}$ sideband manifold that most faithfully reproduces the experimental spectrum is located, while accounting for all sideband orders in the iterative fitting.

Despite that truly isolated half-integer spin-pairs are very rare, spin-pair approximations still provide reliable results provided that the targeted dipolar coupling constant is much larger ( $\gtrsim 10$ times $)$ than those of the other couplings in the system: for example, the directly bonded B-B pair in the bis(catecholato)diboron molecule represents a very strong ${ }^{11}{ }^{11}-{ }^{11} \mathrm{~B}$ dipolar coupling that is $\approx 12$ times larger than its second-largest counterpart. The longer-range intermolecular interactions manifest themselves primarily in the \pm 1 sideband amplitudes that become overemphasized relative to those of the higher-orders; such effects account for most of the deviations between experimental results and those obtained by spin-pair simulations. Yet, an accurate estimate of the large dipolar coupling is still feasible by simply omitting the lowestorder sidebands in the RMS calculation and only evaluating all higher order $( \pm 3, \pm 5, \ldots)$ sideband amplitudes. This effectively amounts to ignoring all weaker dipolar interactions; see Fig. 3 and discussions thereof.

\subsection{Probing spin-cluster sizes}

We next consider the more common scenario of macroscopically large quadrupolar-spin systems encountered in inorganic materials, which implies the absence of well-defined "clusters". Here, analysis of the $2 \mathrm{Q}_{\mathrm{CT}}$ experiment via strategy (B) of Section 2.2 provides information about the number of unique spin sites that contributes to the experimental sideband pattern, the number of which increases with the $2 \mathrm{Q}_{\text {Ст }}$ preparation period. As may be verified both from the CT $2 \mathrm{Q}$ NMR results of the $\mathrm{Na}_{2} \mathrm{SO}_{4}$ and $\alpha-\mathrm{Al}_{2} \mathrm{O}_{3}$ structures in Fig. 6 and 9, there are no $2 \mathrm{Q}_{\mathrm{CT}}$ sideband intensities beyond the lowest $( \pm 1)$ order at $\tau_{\mathrm{exc}} \approx \tau_{\mathrm{exc}}^{\max }$, i.e., when the selected excitation period optimizes the total $2 \mathrm{Q}_{\mathrm{CT}}$ signal amplitude. For such cases, the number of contributing spins is necessarily small and also most difficult to determine, because the discrimination between simulations with distinct cut-off radii is poor.

For increasing $\tau_{\text {exc }}$, the best-fit $R$-value grows and thereby also its associated number of contributing spins. The number of spinning sidebands of significant amplitude is proportional to the product of the $2 \mathrm{Q}_{\mathrm{CT}}$ excitation interval and the effective dipolar interaction of the multi-spin system, i.e., a fictive dipolar coupling constant that approximates the net effect from all spin-pairs in the system; ${ }^{34-36,72,94-96}$ it is dictated by the magnitudes and numbers of all contributing dipolar interactions over a certain $R$-value. Hence, for progressively increased excitation intervals, inspection of the sideband intensitydistributions at the corresponding ratios of $\tau_{\mathrm{exc}} / \tau_{\mathrm{exc}}^{\max }$ gives direct qualitative information about the magnitude of the effective dipolar coupling constant of the probed spin network. For increasing $\tau_{\text {exc }} / \tau_{\text {exc }}^{\max }$ ratio, Table 2 reveals that the observed number of interacting spins grows significantly faster in the $\alpha-\mathrm{Al}_{2} \mathrm{O}_{3}$ structure relative to its $\mathrm{Na}_{2} \mathrm{SO}_{4}$ counterpart, in full accordance with the known higher packing density of $\mathrm{Al}$ in $\alpha-\mathrm{Al}_{2} \mathrm{O}_{3}$ compared to that of $\mathrm{Na}$ in $\mathrm{Na}_{2} \mathrm{SO}_{4}$.

Thus far, the prevailing approach to estimate spin-cluster sizes-"multiple-quantum spin counting",57-64_relies on highorder multiple-quantum coherences (MQC) excitation in stationary or rotating powders comprising multi-spin-1/2 systems. The highest generated MQC order provides a (directly determined) lower bound of $M$ interacting spins present in the "real" cluster. However, only one such attempt at using MQC excitation to count the number of half-integer spins is hitherto reported, then restricted to low-resolution applications in stationary powders. ${ }^{64}$ Our proposed strategy of comparing experimental $2 \mathrm{Q}_{\mathrm{CT}}$-sideband intensities with approximative spin-pair summations constitutes the first general means for estimating the size of a cluster of half-integers spins under MAS conditions. The spin-pair summation strategy is not necessarily restricted to quadrupolar nuclei ${ }^{56}$ we are currently exploring its prospects for spin-1/2 applications.

The main limitation of the present spin-pair summation strategy is its time-consuming numerical analysis. ${ }^{56}$ Yet, despite its feature of providing the spin-cluster sizes indirectly through an (approximate) numerical approach, it exhibits a decisive advantage relative to MQ spin counting in that it avoids the cumbersome high-order MQC excitation. Also noteworthy is that techniques for producing high-order multiple-quantum coherences solely involving the central transitions of halfinteger spins remain to be developed. The current spin-1/2 MQC-excitation strategy is also prone to underestimating the size of large spin-systems due to the impact of NMR relaxation and pulse imperfections, particularly in rotating solids where the progressive high-order MQC generation is slow. Furthermore, also MQ spin-counting data-analyses often resorts to indirect 
estimates of the "real" spin-cluster size by fitting the experimental MQC amplitude envelope to a Gaussian decay; ${ }^{57,58}$ this procedure may be associated with non-negligible uncertainties, as discussed further in ref. 58 and 63.

\section{Conclusions}

In this contribution, we consolidated the application of CT 2Q sideband NMR spectroscopy for estimating internuclear distances within (nearly) isolated pairs of half-integer spin quadrupolar nuclei, and demonstrated that this experimental approach may be combined with numerical analyses to probe multiple-spin effects and estimate the sizes of local clusters of mutually dipolar-coupled quadrupolar nuclei in network structures.

All these aspects were first explored for the case of bis(catecholato)diboron, where each molecule involves one pair of directly bonded boron atoms, while the intermolecular B-B distances are sufficiently long to ensure a nearly isolated ${ }^{11} \mathrm{~B}-{ }^{11} \mathrm{~B}$ spin pair in each molecule. Since the largest coupling is an order of magnitude stronger than its second largest counterpart, we demonstrated the feasibility of determining its magnitude by fitting results from two-spin simulations employing variable dipolar couplings to the experimental results. Considering only the higher oddorder $( \pm 3, \pm 5, \ldots) 2 \mathrm{Q}_{\mathrm{CT}}$ sidebands in the numerical fitting, the B-B internuclear distance was determined as $(168.6 \pm 3) \mathrm{pm}$, i.e., with an uncertainty of $\pm 2 \%$. However, the strong amplitudes of the lowest order $( \pm 1)$ sidebands cannot be reproduced by single spin-pair simulations; they were shown to stem from the longer range intermolecular ${ }^{11} \mathrm{~B}-{ }^{11} \mathrm{~B}$ dipolar interactions. We presented two approaches to account for their contributions, both of which successfully reproduced the entire experimental manifold of $2 \mathrm{Q}_{\mathrm{CT}}$ sidebands: (i) performing computationally expensive multi-spin simulations, or (ii) apply the spin-pair summation approach in ref. 56, in which the complete calculated NMR spectrum is obtained by summation of individual simulations for spin-pairs found within a certain cut-off distance.

Besides justifying the approximative numerical spin-pair simulation protocol by rigorous multi-spin ${ }^{11} \mathrm{~B}-{ }^{11} \mathrm{~B}$ simulations for the case of bis(catecholato)diboron, we further successfully demonstrated it by ${ }^{23} \mathrm{Na}$ and ${ }^{27} \mathrm{Al} \mathrm{NMR}$ on the macroscopic networks of $\mathrm{Na}_{2} \mathrm{SO}_{4}$ and $\mathrm{Al}_{2} \mathrm{O}_{3}$, respectively. For progressively lengthened $2 \mathrm{Q}_{\mathrm{CT}}$ excitation intervals, each respective ${ }^{23} \mathrm{Na}$ and ${ }^{27} \mathrm{Al}$ cluster size may be extracted by locating its corresponding cut-off distance, thereby allowing the monitoring of a growing number of interacting nuclear spins. Altogether, this analysis yields similar information as the MQ spin-counting experiment, ${ }^{57-64}$ but without the cumbersome need to excite highorder MQC, which for MAS applications is hitherto only demonstrated for spin-1/2 systems. ${ }^{59-63}$ However, we emphasize that the $\mathrm{MQ}$ spin-counting technique provides direct estimates of spin-cluster sizes, whereas the $2 \mathrm{Q}_{\mathrm{CT}}$ sideband experiment requires a priori knowledge about the atomic coordinates of the structure for determining the number of interacting spins for the given $2 \mathrm{Q}_{\mathrm{CT}}$ excitation interval.
The approach of analyzing experimental CT $2 \mathrm{Q}$ sideband data by comparison with sums of numerically simulated spin-pair responses may in its present form only be used for discriminating between various proposed structural models through the adherence of their corresponding numerically simulated sideband patterns to the experimental results. Nevertheless, this protocol makes an important dent towards elucidating the dependence of the quadrupolar-spin dynamics on a potentially wide range of structural parameters, such as the positions and local geometries of the quadrupolar spin-sites. As already demonstrated in the context of $2 \mathrm{Q}-1 \mathrm{Q}$ correlation spectroscopy, a spin-pair summation analysis allows for determining the absolute orientations of the electric field gradient (EFG) tensor at each quadrupolar spin-site: ${ }^{56}$ hence, the encoding of distanceinformation in the sidebands from the closely related CT $2 Q$ sideband protocol may be linked to the EFG tensor orientations of the recoupled spins.

Our method prepares the grounds for " $a b$ initio distancemeasurements" by numerical fitting of experimental CT $2 \mathrm{Q}$ spinning sideband data from disordered structures, where direct information about the coordinates of the quadrupolar sites is intractable by other means; for many structures, this technique may constitute the sole option for obtaining (semi-) quantitative information about internuclear distances and spin-cluster sizes. The strategy of analyzing results from $2 \mathrm{Q}$ sideband experiments with a spin-pair summation numerical protocol is generally applicable: we are currently exploring it for spins-1/2 applications to oxide-based inorganic network structures.

\section{Acknowledgements}

The authors would like to thank Igor Moudrakovski, Victor Terskikh and Chris Ratcliffe for helpful discussions. Access to CASTEP was provided by the National Ultrahigh-Field NMR Facility for Solids (Ottawa, Canada), a national research facility funded by the Canada Foundation for Innovation, the Ontario Innovation Trust, Recherche Québec, the National Research Council Canada, and Bruker BioSpin and managed by the University of Ottawa (http://www.nmr900.ca). This work was supported by the National Research Council Canada (NRC), the Swedish Research Council (contract VR-NT 2010-4943) and the Faculty of Sciences at Stockholm University.

\section{References}

1 S. Dusold and A. Sebald, Annu. Rep. NMR Spectrosc., 2000, 41, 185-264.

2 M. H. Levitt, Encyclopedia of Nuclear Magnetic Resonance, Wiley, Chichester, England, 2002, vol. 9, pp. 165-196.

3 M. Edén, eMagRes, 2013, 2, 351-364.

4 V. Ladizhansky, Solid State Nucl. Magn. Reson., 2009, 36, 119-128.

5 N. C. Nielsen, L. A. Strassø and A. B. Nielsen, Solid-State NMR, Springer-Verlag, Berlin/Heidelberg, 2012, vol. 306, pp. 1-45. 
6 I. Schnell, Prog. Nucl. Magn. Reson. Spectrosc., 2004, 45, 145-207.

7 X. Feng, Y. K. Lee, D. Sandström, M. Edén, H. Maisel, A. Sebald and M. H. Levitt, Chem. Phys. Lett., 1996, 257, 314-320.

8 X. Feng, M. Edén, A. Brinkmann, H. Luthman, L. Eriksson, A. Gräslund, O. N. Antzutkin and M. H. Levitt, J. Am. Chem. Soc., 1997, 119, 12006-12007.

9 M. Hong, J. D. Gross and R. G. Griffin, J. Phys. Chem. B, 1997, 101, 5869-5874.

10 M. Edén, A. Brinkmann, H. Luthman, L. Eriksson and M. H. Levitt, J. Magn. Reson., 2000, 144, 266-279.

11 J. C. C. Chan and R. Tycko, J. Am. Chem. Soc., 2003, 125, 11828-11829.

12 V. Ladizhansky, C. P. Jaroniec, A. Diehl, H. Oschkinat and R. G. Griffin, J. Am. Chem. Soc., 2003, 125, 6827-6833.

13 A. Jerschow, Prog. Nucl. Magn. Reson. Spectrosc., 2005, 46, 63-78.

14 S. E. Ashbrook and M. J. Duer, Concepts Magn. Reson., Part A, 2006, 28, 183-248.

15 M. Edén, Solid State Nucl. Magn. Reson., 2009, 36, 1-10.

16 J. V. Hanna and M. E. Smith, Solid State Nucl. Magn. Reson., 2010, 38, 1-18.

17 P. Hartmann, C. Jäger and J. W. Zwanziger, Solid State Nucl. Magn. Reson., 1999, 13, 245-254.

18 M. Nijman, M. Ernst, A. P. M. Kentgens and B. H. Meier, Mol. Phys., 2000, 98, 161-178.

19 M. Baldus, D. Rovnyak and R. G. Griffin, J. Chem. Phys., 2000, 112, 5902-5909.

20 S. Wi, H. Heise and A. Pines, J. Am. Chem. Soc., 2002, 124, 10652-10653.

21 S. Wi, J. W. Logan, D. Sakellariou, J. D. Walls and A. Pines, J. Chem. Phys., 2002, 117, 7024-7033.

22 A. J. Painter and M. J. Duer, J. Chem. Phys., 2002, 116, 710-722.

23 T. G. Ajithkumar and A. P. M. Kentgens, J. Am. Chem. Soc., 2003, 125, 2398-2399.

24 G. Mali, G. Fink and F. Taulelle, J. Chem. Phys., 2004, 120, 2835-2845.

25 M. Edén, H. Annersten and Å. Zazzi, Chem. Phys. Lett., 2005, 410, 24-30.

26 M. Edén, D. Zhou and J. Yu, Chem. Phys. Lett., 2006, 431, 397-403.

27 A. Y. H. Lo and M. Edén, Phys. Chem. Chem. Phys., 2008, 10, 6635-6644.

28 A. Brinkmann, A. P. M. Kentgens, T. Anupõld and A. Samoson, J. Chem. Phys., 2008, 129, 174507.

29 M. Edén and A. Y. H. Lo, J. Magn. Reson., 2009, 200, 267-279.

30 Q. Wang, B. Hu, O. Lafon, J. Trébosc, F. Deng and J. P. Amoureux, J. Magn. Reson., 2009, 200, 251-260.

31 D. Iuga, J. Magn. Reson., 2011, 208, 225-234.

32 M. Edén and L. Frydman, J. Phys. Chem. B, 2003, 107, 14598-14611.

33 M. Edén, J. Grinshtein and L. Frydman, J. Am. Chem. Soc., 2002, 124, 9708-9709.

34 E. Ratai, M. Janssen and H. Eckert, Solid State Ionics, 1998, 105, 25-37.
35 B. A. Gee, Magn. Reson. Chem., 2004, 42, 30-38.

36 J. D. Epping, W. Strojek and H. Eckert, Phys. Chem. Chem. Phys., 2005, 7, 2384-2389.

37 M. J. Duer, Chem. Phys. Lett., 1997, 277, 167-174.

38 N. G. Dowell, S. E. Ashbrook, J. McManus and S. Wimperis, J. Am. Chem. Soc., 2001, 123, 8135-8136.

39 G. Mali and V. Kaučič, J. Magn. Reson., 2004, 171, 48-56.

40 I. Hung, A. P. Howes, T. Anupõld, A. Samoson, D. Massiot, M. E. Smith, S. P. Brown and R. Dupree, Chem. Phys. Lett., 2006, 432, 152-156.

41 M. Edén, J. Magn. Reson., 2010, 204, 99-110.

42 A. Jaworski, B. Stevensson, B. Pahari, K. Okhotnikov and M. Edén, Phys. Chem. Chem. Phys., 2012, 14, 15866-15878.

43 F. A. Perras, I. Korobkov and D. L. Bryce, Phys. Chem. Chem. Phys., 2012, 14, 4677-4681.

44 F. A. Perras, I. Korobkov and D. L. Bryce, CrystEngComm, 2013, 15, 8727-8738.

45 G. Mali, V. Kaučič and F. Taulelle, J. Chem. Phys., 2008, 128, 204503.

46 A. Brinkmann and M. Edén, Can. J. Chem., 2011, 89, 892-899. 47 N. S. Barrow, J. R. Yates, S. A. Feller, D. Holland, S. E. Ashbrook, P. Hodgkinson and S. P. Brown, Phys. Chem. Chem. Phys., 2011, 13, 5778-5789.

48 F. A. Perras and D. L. Bryce, J. Chem. Phys., 2013, 138, 174202.

49 F. A. Perras and D. L. Bryce, J. Am. Chem. Soc., 2013, 135, 12596-12599.

50 H. Geen, J. J. Titman, J. Gottwald and H. W. Spiess, J. Magn. Reson., Ser. A, 1995, 114, 264-267.

51 J. Gottwald, D. E. Demco, R. Graf and H. W. Spiess, Chem. Phys. Lett., 1995, 243, 314-323.

52 R. Graf, D. E. Demco, J. Gottwald, S. Hafner and H. W. Spiess, J. Chem. Phys., 1997, 106, 885-895.

53 U. Friedrich, I. Schnell, S. P. Brown, A. Lupulescu, D. E. Demco and H. W. Spiess, Mol. Phys., 1998, 95, 1209-1227.

54 A. Lupulescu, S. P. Brown and H. W. Spiess, J. Magn. Reson., 2002, 154, 101-129.

55 W. Clegg, M. R. J. Elsegood, F. J. Lawlor, N. C. Norman, N. L. Pickett, E. G. Robins, A. J. Scott, P. Nguyen, N. J. Taylor and T. B. Marder, Inorg. Chem., 1998, 37, 5289-5293.

56 M. Edén, Chem. Phys. Lett., 2009, 470, 318-324.

57 J. Baum, M. Munowitz, A. N. Garroway and A. Pines, J. Chem. Phys., 1985, 83, 2015-2025.

58 S. Lacelle, S. Hwang and B. C. Gerstein, J. Chem. Phys., 1993, 99, 8407-8413.

59 H. Geen, R. Graf, A. S. D. Heindrichs, B. S. Hickman, I. Schnell, H. W. Spiess and J. J. Titman, J. Magn. Reson., 1999, 138, 167-172.

60 N. A. Oyler and R. Tycko, J. Phys. Chem. B, 2002, 106, 8382-8389. 61 M. Edén, Chem. Phys. Lett., 2002, 366, 469-476.

62 P. E. Kristiansen, M. Carravetta, J. D. van Beek, W. C. Lai and M. H. Levitt, J. Chem. Phys., 2006, 124, 234510.

63 G. Teymoori, B. Pahari, E. Viswanathan and M. Edén, J. Magn. Reson., 2013, 236, 31-40.

64 A. J. Dodd and E. R. H. van Eck, Solid State Nucl. Magn. Reson., 2004, 26, 121-131. 
65 J. Haase, M. S. Conradi, C. P. Grey and A. J. Vega, J. Magn. Reson., Ser. A, 1994, 109, 90-97.

66 A. P. M. Kentgens and R. Verhagen, Chem. Phys. Lett., 1999, 300, 435-443.

67 R. Siegel, T. T. Nakashima and R. E. Wasylishen, Chem. Phys. Lett., 2004, 388, 441-445.

68 P. K. Madhu, A. Goldbourt, L. Frydman and S. Vega, Chem. Phys. Lett., 1999, 307, 41-47.

69 Z. Yao, H.-T. Kwak, D. Sakellariou, L. Emsley and P. J. Grandinetti, Chem. Phys. Lett., 2000, 327, 85-90.

70 M. Carravetta, M. Edén, X. Zhao, A. Brinkmann and M. H. Levitt, Chem. Phys. Lett., 2000, 321, 205-215.

71 G. Bodenhausen, H. Kogler and R. R. Ernst, J. Magn. Reson., 1984, 58, 370-388.

72 A. Brinkmann, M. Edén and M. H. Levitt, J. Chem. Phys., 2000, 112, 8539-8554.

73 M. Edén, Concepts Magn. Reson., Part A, 2003, 18A, 1-23.

74 N. C. Nielsen, H. Bildsøe, H. J. Jakobsen and M. H. Levitt, J. Chem. Phys., 1994, 101, 1805-1812.

75 D. States, R. A. Haberkorn and D. J. Ruben, J. Magn. Reson., 1982, 48, 286-292.

76 D. Marion, M. Ikura, R. Tschudin and A. Bax, J. Magn. Reson., 1989, 85, 393-399.

77 M. Bak and N. C. Nielsen, J. Magn. Reson., 2000, 147, 296-330.

78 S. K. Zaremba, SIAM Rev., 1968, 10, 303-314.

79 H. Conroy, J. Chem. Phys., 1967, 47, 5307-5318.

80 V. B. Cheng, H. H. Suzukawa Jr. and M. Wolfsberg, J. Chem. Phys., 1973, 59, 3992-3999.

81 S. J. Clark, M. D. Segall, C. J. Pickard, P. J. Hasnip, M. I. J. Probert, K. Refson and M. C. Payne, Z. Kristallogr., 2005, 220, 567-570.

82 C. J. Pickard and F. Mauri, Phys. Rev. B: Condens. Matter Mater. Phys., 2001, 63, 245101.
83 J. R. Yates, C. J. Pickard and F. Mauri, Phys. Rev. B: Condens. Matter Mater. Phys., 2007, 76, 024401.

84 M. Profeta, F. Mauri and C. J. Pickard, J. Am. Chem. Soc., 2003, 125, 541-548.

85 J. P. Perdew, K. Burke and M. Ernzerhof, Phys. Rev. Lett., 1996, 77, 3865-3868.

86 F. C. Hawthorne and R. B. Ferguson, Can. Mineral., 1975, 13, 181-187.

87 D. S. J. Lewis and H. D. Flack, Acta Crystallogr., Sect. A: Cryst. Phys., Diffr., Theor. Gen. Cryst., 1982, 38, 733-739.

88 G. P. Holland, B. R. Cherry and T. M. Alam, J. Magn. Reson., 2004, 167, 161-167.

89 G. A. F. Seber and C. J. Wild, Nonlinear Regression, WileyInterscience, Hoboken, USA, 2003.

90 E. M. L. Beale, J. R. Stat. Soc. Ser. B, 1960, 22, 41-88.

91 Y. Ishii, T. Terao and S. Hayashi, J. Chem. Phys., 1997, 107, 2760-2774.

92 R. E. Wasylishen, Indirect Nuclear Spin-Spin Coupling Tensors, eMagRes, 2007, DOI: 10.1002/9780470034590.emrstm0237.

93 L. Frydman and J. S. Harwood, J. Am. Chem. Soc., 1995, 117, 5367-5368.

94 C. Filip, S. Hafner, I. Schnell, D. E. Demco and H. W. Spiess, J. Chem. Phys., 1999, 110, 423-440.

95 V. E. Zorin, S. P. Brown and P. Hodgkinson, Mol. Phys., 2006, 104, 293-304.

96 J. S. auf der Günne, J. Magn. Reson., 2006, 180, 186-196.

97 R. K. Harris, E. D. Becker, S. M. Cabral de Menezes, P. Granger, R. E. Hoffman and K. W. Zilm, Pure Appl. Chem., 2008, 80, 59-84.

98 G. E. Hubert Koller, A. P. M. Kentgens and J. Sauer, J. Phys. Chem., 1994, 98, 1544-1551.

99 T. Vosegaard and H. J. Jakobsen, J. Magn. Reson., 1997, 128, 135-137. 